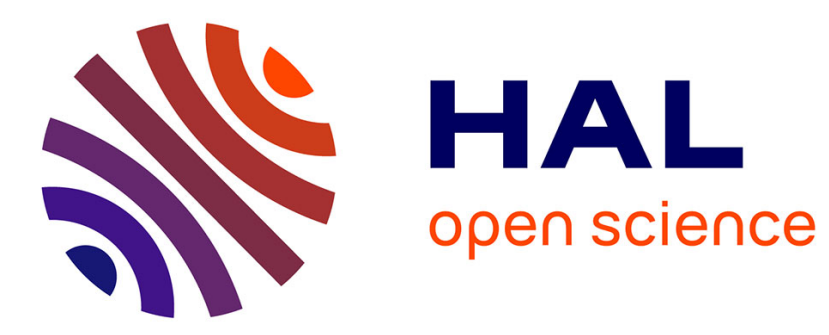

\title{
Functional renormalisation group in a finite volume
}

\author{
Leonard Fister, Jan Martin Pawlowski
}

\section{To cite this version:}

Leonard Fister, Jan Martin Pawlowski. Functional renormalisation group in a finite volume. Physical Review D, 2015, 92 (7), pp.076009. 10.1103/PhysRevD.92.076009 . cea-01332610

\section{HAL Id: cea-01332610 https://hal-cea.archives-ouvertes.fr/cea-01332610}

Submitted on 16 Jun 2016

HAL is a multi-disciplinary open access archive for the deposit and dissemination of scientific research documents, whether they are published or not. The documents may come from teaching and research institutions in France or abroad, or from public or private research centers.
L'archive ouverte pluridisciplinaire HAL, est destinée au dépôt et à la diffusion de documents scientifiques de niveau recherche, publiés ou non, émanant des établissements d'enseignement et de recherche français ou étrangers, des laboratoires publics ou privés. 


\title{
Functional renormalisation group in a finite volume
}

\author{
Leonard Fister ${ }^{1}$ and Jan M. Pawlowski ${ }^{2,3}$ \\ ${ }^{1}$ Institut de Physique Théorique, CEA Saclay, F-91191 Gif-sur-Yvette, France. \\ ${ }^{2}$ Institut für Theoretische Physik, Universität Heidelberg, Philosophenweg 16, 69120 Heidelberg, Germany \\ ${ }^{3}$ ExtreMe Matter Institute EMMI, GSI Helmholtzzentrum für \\ Schwerionenforschung mbH, Planckstr. 1, D-64291 Darmstadt, Germany
}

\begin{abstract}
We study a $\phi^{4}$-theory at finite temperature in a finite volume. Quantum, thermal and volume fluctuations are treated with the functional renormalisation group. Specifically, we focus on the interplay of temperature and length scales driving the system. We find that thermodynamical observables at finite volume such as the pressure approach the infinite volume limit similarly to that of the vanishing temperature limit.

We also advance the functional renormalisation group method at finite volume. In particular, we identify requirements for suitable regulators that admit the exponential thermal and finite volume decay properties.
\end{abstract}

PACS numbers: 11.10.Hi, 11.10.Wx, 11.15.Tk

\section{INTRODUCTION}

Finite volume/finite size effects play or may play an important rôle in systems ranging from the physics of ultracold atom clouds or optical lattices over condensed matter systems, multi-layer systems to heavy-ion collisions where the interaction region is relatively sharply bounded, though expanding. On the theoretical side, lattice methods or other theoretical approaches with spacetime coarse graining are subject to finite volume effects due to finite number of lattice or grid points.

In an Euclidean field theory finite volume effects are described similarly to that of thermal fluctuations, hence being related to a wealth of interesting phenomena and showing characteristic scaling laws. In particular, systems with a second order phase transition lose this property in a finite volume and show finite size scaling. Prominent questions in this context concern the approach to the infinite volume limit and the continuum limit in lattice theories, the characteristics of the finite volume scaling and the appropriate extraction of thermodynamical observables in a given approach.

It is well-known from systems at finite temperature that thermal effects both show a characteristic exponential decay with the masses of the system at hand, as well as a characteristic polynomial decay with momenta due to thermal contact terms. The latter contact terms also play a pivotal rôle in thermodynamical relations. Moreover, the strength of such contribution is only computed with the correct exponential thermal decay. It is this peculiar combination of exponential and polynomial suppression that requires a quantitative control in nonperturbative approaches.

In the present work, we discuss finite temperature and finite volume effects within the functional renormalisation group (FRG) approach, see e.g. 1 15 . Here, we extend the discussions and approach set up in Refs. 6 10 with a special emphasis on the interplay of the scaling with temperature $T$, volume (box length $L$ ), and with the infrared cutoff scale $k$ in the FRG approach. This is worked out at the example of a real $\phi^{4}$-theory. Only if all these scalings are taken into account quantitatively one can expect to have access, in particular, to thermodynamical observables and the equation of state.

A related functional approach is given by DysonSchwinger equations (DSEs) at finite temperature and a finite volume, see e.g. 11-14, if the infrared cutoff scale $k$ is substituted with the ultraviolet initial scale $\Lambda$, where the flow is initiated. The latter can be identified with the ultraviolet momentum cutoff in the DSE framework.

The work is organised as follows. In Section III we discuss the FRG framework for $\phi^{4}$-theory, including its properties and limits of the current simple $\phi^{4}$ approximation. In Section III the formulation of the FRG at finite temperature and volume is introduced in a selfcontained way, with a special emphasis on the fate of long range correlations and the (non-)existence of condensates. In Section [V] we contrast thermodynamical quantities in an infinite and finite volume, and their FRG evolution. Special attention is paid to the relation of the chosen cutoff procedure (regulator function) and the decay behaviour of thermal and finite volume effects for large cutoff scales. In Section $\mathrm{V}$ we extend the decay analysis to the full dynamics. We also present numerical results for the pressure, the dynamical mass and $\phi^{4}$-coupling for finite volume and temperature adapted regulators.

\section{II. $\phi^{4}$-THEORY AND THE FUNCTIONAL RENORMALISATION GROUP}

We study finite volume effects at the simple example of a one-component scalar field theory. Its classical action is given by

$$
S[\phi]=\int \mathrm{d}^{4} x\left[\frac{1}{2}\left(\partial_{\mu} \phi(x)\right)^{2}+V^{\mathrm{cl}}(\rho(x))\right], \quad \rho \equiv \frac{1}{2} \phi^{2},
$$


with the standard kinetic term and a potential

$$
V^{\mathrm{cl}}(\rho)=\frac{\lambda}{2}(\rho-\kappa)^{2}-\frac{\lambda}{2}\left(\rho_{0}-\kappa\right)^{2}, \quad \rho_{0}=\max (0, \kappa) .
$$

The potential $V^{\mathrm{cl}}$ comprises the mass term and the $\phi^{4}$ potential with coupling $\lambda$. The minimum of the potential is at $\rho_{0}=\max (0, \kappa)$. The potential only depends on $\phi^{2}$, thus the theory is $Z_{2}$-invariant under $\phi \rightarrow-\phi$. The parameterisation of the potential in $(2)$ has the normalisation $V^{\mathrm{cl}}\left(\rho_{0}\right)=0$ at the minimum $\rho_{0}$.

\section{A. Functional renormalisation group}

The functional renormalisation group (FRG) is a functional continuum approach that includes nonperturbative effects. In this Section we highlight those aspects which are relevant for this work.

The idea of the FRG is based on Wilson's idea of integrating out fluctuations momentum-shell-wise. In practice, this is done by starting at a microscopic scale $\Lambda$, where the theory is defined via its classical action $S[\phi]$.

In this work, we consider a field theory for a (onecomponent) scalar $\phi$. Fluctuations from larger distance scales with respect to the microscopic scale, i.e. energies lower than the renormalisation group (RG) infrared scale $k$, are suppressed by adding a regulator term, $R_{k}(p)$, which leaves the ultraviolet unchanged but serves as an effective infrared mass $\sim k^{2}$. This allows to integrate fluctuations which are of the order of this mass. By variation of the scale from microscopic to macroscopic values fluctuations from all scales can be taken into account successively. Within this procedure, one smoothly interpolates between the classical action at large cutoff scales $k$ and the full quantum effective action $\Gamma[\phi]$ at vanishing cutoff scale, $k=0$. This is achieved in terms of an integrodifferential equation, the Wetterich equation, [15,

$$
\partial_{t} \Gamma_{k}[\phi]=\frac{1}{2} \int_{q} \frac{1}{\Gamma^{(2)}[\phi]+R_{k}} \partial_{t} R_{k}(q),
$$

with the standard abbreviation $\int_{q}=\int \mathrm{d}^{4} q /(2 \pi)^{4}$, and $t=\ln k / \Lambda$ with some reference scale $\Lambda$. The schematic notation on the right hand side of (3) stands for integration of the full, non-perturbative diagonal part of the propagator $1 /\left(\Gamma^{(2)}+R_{k}\right)(p, q)$ and the derivative of the regulator $R_{k}(q)$ over the loop momentum $q$. The initial condition for $(3)$ is given by $\Gamma_{\Lambda}[\phi]=S[\phi]$. For general $k$, the effective action $\Gamma_{k}[\phi]$ describes the full quantum effective action for momentum scales larger than $k$, and lacks the quantum fluctuations of momentum scale smaller than $k$. In the limit $k \rightarrow 0$ it turns into the full quantum effective action $\Gamma[\phi]$.

The regulator implements the shell-wise integration of fluctuations described above, i.e. suppressing the infrared while leaving the ultraviolet unmodified. Within these restrictions it can be chosen freely. In the following, we

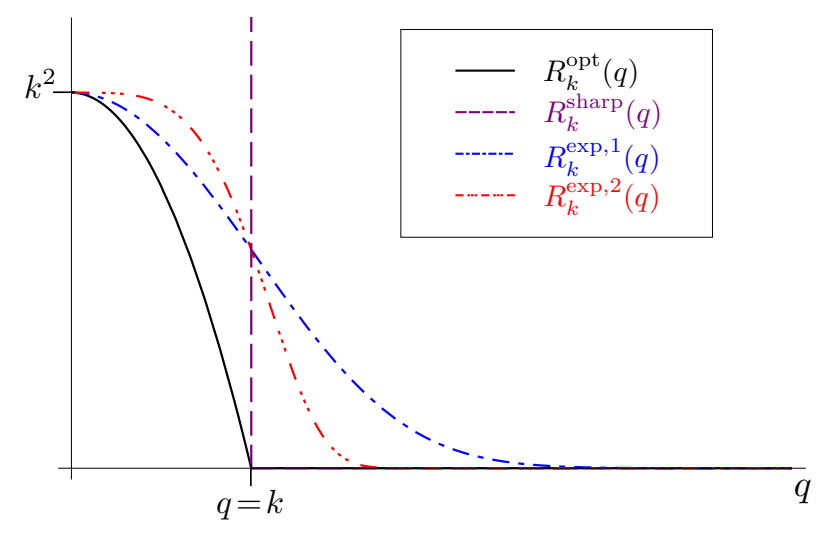

FIG. 1: Different regulator functions, see (4), (5) and (6).

study various common choices like the exponential regulator,

$$
R_{k}^{\exp , \mathrm{m}}(q)=q^{2} \frac{\left(q^{2} / k^{2}\right)^{m-1}}{e^{\left(q^{2} / k^{2}\right)^{m}-1}}
$$

where the parameter $m$ controls the sharpness of suppression above the scale $k$, the flat regulator [16],

$$
R_{k}^{\mathrm{flat}}(q)=\left(k^{2}-q^{2}\right) \Theta\left(k^{2}-q^{2}\right)
$$

which is optimised within the lowest order of a derivative expansion, see [16 18], and the sharp regulator,

$$
R_{k}^{\text {sharp }}(q)=k^{2}\left(\frac{1}{\Theta\left(k^{2}-q^{2}\right)}-1\right) .
$$

The sharp regulator implements the standard ultraviolet momentum regularisation within Dyson-Schwinger equations, as well as in perturbation theory. There, loop momenta $q^{2}$ are cut at an ultraviolet scale $\Lambda$ with $q^{2} \leq \Lambda^{2}$. Integrating the flow with the sharp regulator from an ultraviolet initial scale $k=\Lambda$ to $k=0$ precisely leads to such loop integrals: in this case the flow gives the standard BPHZ-type renormalisation. Other regulators give a generalised BPHZ-type renormalisation, see [18, in particular the regulators (4) do not spoil the analytic properties of the integrands for finite momenta. As we shall see, the non-analyticity of the flat regulator is already causing large intricacies for the flow of thermodynamical observables. These intricacies get enhanced by the stronger non-analyticity of the sharp regulator.

The regulators (4), (5), (6) are shown in Fig. 1. Note that the regulator does not need to depend on both, frequencies and spatial momenta. In fact, in the context of thermal field theory it is convenient to regulate spatial momenta only, $q^{2} \rightarrow \vec{q}^{2}$ in the regulators defined in (4) - (6), because in this case Matsubara frequency sums 
can often be performed analytically. We will elaborate on this in section IV, where we focus also on the suitability of regulators from additional constraints imposed by finite temperature or volume.

\section{B. Local potential approximation and flow of the effective potential}

For large cutoff scales $k \rightarrow \Lambda$, the effective action $\Gamma_{k}$ tends towards the classical action $S$ in (1) with $\Lambda$ dependent parameters. In particular the effective potential $V_{k}(\rho)$ tends towards the (bare) classical potential, $V_{\Lambda}=V_{\mathrm{cl}}$. Here, the effective potential $V_{k}$ is defined as the effective action $\Gamma_{k}$ evaluated for static (constant) fields $\rho_{c}$

$$
V_{k}\left(\rho_{c}\right) \equiv \frac{\Gamma_{k}\left[\rho=\rho_{c}\right]}{\mathcal{V}_{4 \mathrm{~d}}}, \quad \text { with } \quad \mathcal{V}_{4 \mathrm{~d}}=\prod_{i=\mu}^{4} \int_{0}^{L_{\mu}} d x_{\mu}
$$

In (7) the trivial volume factor is removed. The effective action also contains higher derivative terms which are dropped here in the spirit of a low energy derivative expansion or local potential approximation (LPA). In this approximation, the full effective action reads

$$
\Gamma_{k}[\phi]=\int \mathrm{d}^{4} x\left(\frac{1}{2}\left(\partial_{\mu} \phi\right)^{2}+V_{k}(\rho)\right),
$$

Within the LPA approximation (8), the $k$-dependent full propagator is given by

$$
G_{k}(q, \rho)=\frac{1}{q^{2}+m_{k}^{2}(\rho)+R_{k}(q)},
$$

with the $k$ - and field-dependent mass function

$$
m_{k}^{2}(\rho)=V_{k}^{\prime}(\rho)+2 \rho V_{k}^{\prime \prime}(\rho) .
$$

The primes indicate derivatives with respect to $\rho$. The effective potential $V_{k}(\rho)$ can be expanded in powers of $\left(\rho-\rho_{0}\right)$ about the equation of motion (EoM) for static fields,

$$
\left.\partial_{\phi} V\right|_{\phi=\sqrt{2 \rho_{0}}}=0
$$

This leads to

$$
\begin{aligned}
V_{k}(\rho)= & V_{k}\left(\rho_{0}\right)-\frac{\lambda}{2}\left(\rho_{0}-\kappa\right)^{2}+\frac{\lambda}{2}(\rho-\kappa)^{2} \\
& +\sum_{n=3}^{\infty} \frac{\lambda_{n}}{n !}\left(\rho-\rho_{0}\right)^{n},
\end{aligned}
$$

with the relation between $\rho_{0}$ and $\kappa$ from (2) and $V_{\Lambda}\left(\rho_{0}\right)=$ 0 . The first line comprises the normalisation of the effective potential and the $k$-dependent counterpart of the classical potential including fluctuation effects for $\kappa$ and $\lambda$, while the second line comprises the fluctuation- induced higher order scatterings. In this expansion the $k$ dependent analogues of the power-counting relevant classical parameters $\lambda, \kappa$ are determined by

$$
\lambda_{k}\left(\rho_{0, k}-\kappa_{k}\right)=V_{k}^{\prime}\left(\rho_{0, k}\right), \quad \lambda_{k}=V_{k}^{\prime \prime}\left(\rho_{0, k}\right)
$$

where we made the $k$-dependence explicit. The mass function $m_{k}^{2}(\rho)$, evaluated on the EoM, reads

$$
m_{k}^{2}=m_{k}^{2}\left(\rho_{0, k}\right)=\lambda_{k}\left(3 \rho_{0, k}-\kappa_{k}\right),
$$

by using the definition of $\kappa$ in 2 . Vanishing $\kappa$ signals the phase transition. For large cutoff scales $k \rightarrow \Lambda$, the effective potential tends towards the classical potential $V_{\mathrm{cl}}$ given in (2).

The flow equation for the effective potential is easily derived from $(3)$ and reads

$$
\dot{V}_{k}(\rho)=\frac{1}{2} \int_{q} G(q, \rho) \dot{R}_{k}(q),
$$

with $G(q, \rho)$ defined in (9), and the dot indicates the dimensionless derivative with respect to the RG scale, i.e. $\dot{V}_{k}=\partial_{t} V_{k}$. The flow of the effective potential, 15$)$ depends on $V^{\prime}$ and $V^{\prime \prime}$ via the mass function $m_{k}^{2}(\rho)$, 10 in the propagator. By taking the first and second derivative of (15) with respect to $\rho$ in (1) we find

$$
\begin{aligned}
\partial_{\rho} \dot{V}_{k}(\rho) & =\frac{1}{2} \int_{q} \frac{\partial}{\partial \rho} G_{k}(q, \rho) \dot{R}_{k}(q) \\
& =-\frac{1}{2}\left(m_{k}^{2}(\rho)\right)^{\prime} \int_{q} G_{k}^{2}(q, \rho) \dot{R}_{k}(q)
\end{aligned}
$$

and

$$
\begin{aligned}
\partial_{\rho}^{2} \dot{V}_{k}(\rho)= & {\left[\left(m_{k}^{2}(\rho)\right)^{\prime}\right]^{2} \int_{q} G_{k}^{3}(q, \rho) \partial_{t} R_{k}(q) } \\
& -\frac{1}{2}\left(m_{k}^{2}(\rho)\right)^{\prime \prime} \int_{q} G_{k}^{2}(q, \rho) \dot{R}_{k}(q) .
\end{aligned}
$$

Eq. (16) and (17) depend on first and second $\rho$-derivatives of the mass function, whose flows can be obtained by further derivatives of the flow equation (17) with respect to $\rho$.

\section{C. $\phi^{4}$-approximation}

The partial differential equation (15) can be solved within various methods. Here, we resort to a Taylor expansion about the minimum $\rho_{0}$. Then, evaluated at $\rho_{0}$, (15)-(17) are the first three of an infinite hierarchy of ordinary coupled differential equations for the couplings $\kappa, \lambda, \lambda_{n \geq 3}$ defined in 12 . At the initial scale $\Lambda$ we have $\lambda_{n, \Lambda}=0$ for $n \geq 3$. Using the parameterisation 12 we get for the left hand side of the flow of the effective potential 
(15),

$$
\begin{aligned}
\partial_{t} V(\rho)= & \dot{V}\left(\rho_{0}\right)-\dot{\rho}_{0}\left[V^{\prime}(\rho)-V^{\prime}\left(\rho_{0}\right)\right]+\lambda\left(\dot{\rho}_{0}-\dot{\kappa}\right)\left(\rho-\rho_{0}\right) \\
& +\frac{\dot{\lambda}}{2}(\rho-\kappa)^{2}-\frac{\dot{\lambda}}{2}\left(\rho_{0}-\kappa\right)^{2}+\sum_{n \geq 3} \frac{\dot{\lambda}_{n}}{n !}\left(\rho-\rho_{0}\right)^{n},
\end{aligned}
$$

leading to

$$
\dot{V}^{\prime}\left(\rho_{0}\right)=-\lambda \dot{\kappa}, \quad \dot{V}^{\prime \prime}\left(\rho_{0}\right)=\dot{\lambda}-\dot{\rho}_{0} \lambda_{3} .
$$

The $\lambda_{3}$ term in (19) signals the $k$-dependence of the expansion point and feeds into the flow of $\lambda$. This exemplifies the destabilising backreaction of the higher couplings on the flow of the lower couplings in the presence of a flowing expansion point, and can be avoided with a $k$-independent expansion point, [19]. In the present work we drop the higher couplings also for $k<\Lambda$, that is $\lambda_{n \geq 3} \equiv 0$ and approximate

$$
V_{k}(\rho) \approx V_{k}^{(0)}\left(\rho_{0}\right)+\left(\rho-\rho_{0}\right) V_{k}^{\prime}\left(\rho_{0}\right)+\frac{1}{2}\left(\rho-\rho_{0}\right)^{2} V_{k}^{\prime \prime}\left(\rho_{0}\right),
$$

with (13). This is simply the first line in 12 . The rapid convergence of this expansion in $O(N)$-models at infinite volume in general dimensions has been well-studied for example within the computation of critical exponents, see e.g. 20 23. This approximation also works well for low-energy effective models for QCD with mesonic degrees of freedom at vanishing density and finite temperature [19. In turn, at finite density and finite temperature higher powers in $\rho$, further couplings and momentum dependences are required for quantitative statements, for details see Ref. [19]. This concerns in particular the existence and location of a critical end point (CEP) in the phase diagram. Then one either resorts to a $k$-independent expansion point or relies on global techniques for solving partial differential equations, see e.g. [2, 24].

Here, we are interested in structural results and we only keep the relevant parameters $\kappa$ and $\lambda$ for the sake of simplicity. Then the set of flow equations (15)-(17) is closed. In this approximation, we have access to the flowing free energy density $f_{k}$, the mass $m_{k}^{2}$, see 114 , and the coupling $\lambda_{k}$ with

$$
f_{k} \equiv V_{k}\left(\rho_{0}\right)=\frac{\Gamma_{k}\left[\rho_{0}\right]}{\mathcal{V}_{4 \mathrm{~d}}},
$$

and

$$
m_{k}^{2}=V_{k}^{\prime}\left(\rho_{0}\right)+2 \rho_{0} V_{k}^{\prime \prime}\left(\rho_{0}\right), \quad \lambda_{k} \equiv V_{k}^{\prime \prime}\left(\rho_{0}\right) .
$$

The two parameters in 22 can be determined from the coupled set of flow equations for $\partial_{\rho} \dot{V}_{k}\left(\rho_{0}\right)$ and $\partial_{\rho}^{2} \dot{V}_{k}\left(\rho_{0}\right)$. The flow of the free energy density $f_{k}$ in $(21)$ only depends on $\kappa_{k}$ and $\lambda_{k}$, and can be integrated separately with the solution for $\kappa_{k}$ and $\lambda_{k}$.

In the current $\phi^{4}$-approximation the mass function and its derivatives are given by

$$
m_{k}^{2}(\rho)=\lambda_{k}\left(3 \rho-\kappa_{k}\right), \quad m_{k}^{2^{\prime}}=3 \lambda_{k}, \quad m_{k}^{2^{\prime \prime}} \equiv 0 .
$$

In summary, this leads to the closed system of flow equations for $f_{k}, \kappa_{k}$ and $\lambda_{k}$, with

$$
\begin{aligned}
& \dot{f}_{k}=\frac{1}{2} \int_{q} \dot{R}_{k}(q) G_{k}(q), \\
& \dot{\kappa}_{k}=\frac{3}{2} \int_{q} \dot{R}_{k}(q) G_{k}^{2}(q), \\
& \dot{\lambda}_{k}=9 \lambda_{k}^{2} \int_{q} \dot{R}_{k}(q) G_{k}^{3}(q) .
\end{aligned}
$$

Note that all flows are positive and therefore leading to decreasing functions $f_{k}, \kappa_{k}$ and $\lambda_{k}$. In summary this results in an increasing mass. This reflects the property that bosonic flows are symmetry-restoring. Note also that the reduced system of equations for the mass and the coupling is already closed. The flow of $f_{k}$ only depends on $m_{k}=\lambda_{k}\left|\kappa_{k}\right|$. The above flows integrations over the four-momentum $q$ : for finite extent in either temporal or spatial direction the integration (partly) turns into summation over discrete modes. We elaborate on these modifications in section III

\section{FINITE TEMPERATURE \& VOLUME}

At finite temperature and/or in a finite volume, some of the space-time directions only have finite extent,

$$
x_{0} \in[0,1 / T], \quad \text { and/or } x_{i} \in\left[0, L_{i}\right] .
$$

Note that in the current Euclidean formulation there is no technical difference between the spatial and temporal directions. One might as well interpret a finite extent in the temporal direction as a theory at vanishing temperature and one compact spatial direction with $L_{0}=1 / T$. A finite extent in a given direction with periodic boundary conditions (for bosons, $\left.\phi\left(x+L_{\mu}\right)=\phi(x)\right)$ ) only allows for plane waves that are periodic under shifts $x_{\mu} \rightarrow x_{\mu}+L_{\mu}$, that are $\exp \left(i 2 \pi n x_{\mu} / L_{\mu}\right)$ with $n \in \mathbb{Z}$. This entails that frequencies and momenta are discrete,

$$
p_{\mu}=2 \pi n \frac{1}{L_{\mu}}, \quad \text { with } \quad n \in \mathbb{Z} \quad \text { and } \quad T=\frac{1}{L_{0}} .
$$

In the case of finite temperature these are the Matsubara frequencies. Accordingly, spatial momentum integrations turn into sums over spatial Matsubara modes. The compactification of a coordinate $x$ related to the momentum $p$ to the interval $x \in[0, L]$ with periodic boundary con- 
ditions leads to

$$
\frac{1}{2 \pi} \int_{-\infty}^{\infty} \mathrm{d} q I\left(q^{2}\right) \stackrel{L<\infty}{\rightarrow} \frac{1}{L} \sum_{n=-\infty}^{\infty} I\left(\left(\frac{2 \pi n}{L}\right)^{2}\right) .
$$

This is trivially extended to higher dimensions.

In the case of finite temperature the temporal direction $x_{0}$ is compactified to the range $x_{0} \in[0,1 / T]$ with periodic boundary conditions for bosons. For fermions one has anti-periodic boundary conditions. For infinite spatial extent, momentum integrations have the form

$$
\int_{q} I\left(q^{2}\right) \stackrel{T>0}{\longrightarrow} T \sum_{n_{0}=-\infty}^{\infty} \int_{\vec{q}} I\left(\left(2 \pi T n_{0}\right)^{2}+\vec{q}^{2}\right),
$$

with the spatial momentum integration $\int_{\vec{q}}=\int \mathrm{d}^{3} q /(2 \pi)^{3}$, and a sum over the Matsubara modes $2 \pi T n_{0}$.

In the case of finite temperature and finite spatial extent $L$ we are left with sums over discrete modes in both temporal and spatial directions, respectively, viz.

$$
\begin{aligned}
\int_{q} I\left(q^{2}\right) \underset{L<\infty}{\stackrel{T>0}{\longrightarrow}} & \\
& \frac{T}{L^{3}} \sum_{\substack{n_{\mu}=-\infty \\
\mu=0,1,2,3}}^{\infty} I\left(4 \pi^{2}\left[T^{2} n_{0}^{2}+\frac{n_{1}^{2}+n_{2}^{2}+n_{3}^{2}}{L^{2}}\right]\right),
\end{aligned}
$$

with $n_{1}, n_{2}$ and $n_{3}$ label the discrete spatial modes in the cubic volume of edge length $L$.

\section{A. Range of finite temperature \& volume effects}

Finite extent, (25), seemingly induces a dimensional reduction of the theory in the presence of finite temperature and/or finite volume: for large temperature/small volume only the zero mode of the sum contributes, leaving only the integration/sum over the other momentum directions. This dimensional reduction is well-known at finite temperature. There it is commonly formulated that a quantum field theory in $3+1$ (or other dimensions) is dimensional reduced to its 3-dimensional counterpart for sufficiently large temperature. However, strictly speaking this only holds for momentum scales $p / T \ll 1$. There, however, it is valid for all temperatures. In turn, for $p / T \gg 1$ the dimensional reduction does not take place. This is reflected in the fact that the renormalisation can be chosen to be temperature-independent.

The co-existence and interaction of these two momentum regimes for all temperatures and/or finite lengths has the direct consequence that we see both, an exponential suppression of thermal and/or finite volume effects with the physical mass scales of the theory as well as the standard polynomial suppression of large momentum modes already present in perturbation theory:

Exponential decay: In finite temperature field theory thermal correlations decay exponentially with the mass gap $m_{\text {gap }}$ of the theory, which can be formalised as

$$
\lim _{\frac{m_{\text {gap }}}{T} \rightarrow \infty}\left|\frac{\mathcal{O}_{n}^{T}-\mathcal{O}_{n}^{T=0}}{\mathcal{O}_{n}^{T=0}}\right| \propto A\left(\frac{m_{\text {gap }}}{T}\right) \exp \left(-\frac{m_{\text {gap }}}{T}\right),
$$

for correlation functions

$$
\mathcal{O}_{n}^{T}=\left\langle\phi\left(x_{1}\right) \cdots \phi\left(x_{n}\right)\right\rangle_{T},
$$

in the absence of further scales. If the latter ones are present they lead to further dependencies on their dimensionless ratios in $A$. The function $A(y)$ is a rational function of the argument $y=m_{\text {gap }} / T$, and the exponential suppression with $\exp \left(-m_{\text {gap }} / T\right)$ can be readily computed from one loop thermal perturbation theory. Evidently, similar expressions hold for the finite volume correlations, with the identification $T \rightarrow 1 / L_{i}$ in 30 . For potentially vanishing correlation functions the denominator of 30 should be substituted with $\left|\mathcal{O}_{n}^{T}\right|+\left|\mathcal{O}_{n}^{T=0}\right|$ for the sake of definiteness. For the correlation functions studied in the present work this is not necessary.

Polynomial decay: Note that 30 does not imply an exponential suppression of thermal corrections in momentum space with momenta proportional to $\exp (-$ const. $p / T)$. In momentum space, correlation functions show a subleading momentum behaviour with temperature-dependent coefficients that is only suppressed by powers of $p^{2}$. For the sake of simplicity, we consider the symmetric point $p_{i}^{2}=p^{2}$ for all $i=1, \ldots, n$ and obtain

$$
\lim _{\frac{p}{T} \rightarrow \infty}\left|\frac{\mathcal{O}_{n}^{T}-\mathcal{O}_{n}^{T=0}}{\mathcal{O}_{n}^{T=0}}\right| \propto B\left(\frac{p}{T}\right) \rightarrow 0,
$$

with a rational function $B(p / T)$ which decays polynomially for $p / T \rightarrow \infty$.

For the two-point correlation functions, the propagator $G(p)$, this is known as the Tan-contact term in e.g. the context of ultracold atoms 25 27. Within the FRG these terms have been discussed in [6, 8, 28, for YangMills theory and ultracold atoms, respectively. In 6 6 8 a detailed analysis of the diagrammatic origin of this behaviour was provided. This also entails that the coefficients of this decay come from thermal fluctuations and their values hinge crucially on the correct implementation of the exponential decay in 32 . Hence, even though these terms have a polynomial decay, the correct computation of the prefactor $C_{T}$ relies on the exponential suppression 30 with the physical mass scales of the theory. For the propagator we have

$$
\lim _{\frac{|\vec{p}|}{T} \rightarrow 0}\left|\frac{G^{T}(0, \vec{p})-G^{T=0}(0, \vec{p})}{G^{T=0}(0, \vec{p})}\right| \propto C_{T}\left(\frac{T}{|\vec{p}|}\right)^{4},
$$

with a temperature-dependent prefactor $C_{T}$ (in the presence of other scales), the Tan-contact. Importantly, the 
Tan-contact is related to e.g. the equation of state as well as the density. Hence, it is expected that only an approach that is able to deal with both the exponential suppression and the polynomial terms enables us to compute these quantities.

\section{B. Flow equation at finite temperature \& volume} form

The flow equation for the effective potential takes the

$$
\dot{V}_{k}(\rho)=\frac{T}{2 L^{3}} \sum_{n_{\mu} \in \mathbb{Z}} G_{k}(q, \rho) \dot{R}(q), \quad \text { with } \quad q_{\mu}=2 \pi n_{\mu} \frac{1}{L_{\mu}} .
$$

Note that the effects of finite volume are qualitatively similar in other dimensions $(d-1)+1$. Thus, for the purpose of this work, we restrict ourselves to $(3+1)$ dimensions. In the following, we discuss results obtained in the $\phi^{4}$-approximation for flows at both finite temperature and finite volume. These results are obtained by integrating the flows (containing loop integrations $29 \mathrm{p}$ ), 24, over the RG scale $k$ from $\Lambda$ to 0 .

In the current FRG setup the dimensional reduction discussed in the last section IIIA is apparent from the flow. To begin with, at finite temperature and $k / T \ll 1$ the flow only receives contributions from the Matsubara zero mode (for regulators that decay sufficiently fast for frequencies $\left.q_{0} / T \ll 1\right)$. Consequently, in this regime the flow reads

$$
\dot{V}_{k \ll T}(\rho) \rightarrow \frac{T}{2} \int_{\vec{q}} \frac{\dot{R}_{k}\left(q_{0}=0,|\vec{q}|\right)}{\vec{q}^{2}+R_{k}\left(q_{0}=0,|\vec{q}|\right)+m_{k}(\rho)} .
$$

The prefactor $T$ can be absorbed with an appropriate rescaling of $V_{k}, \rho$, leading to dimensionally reduced flows for

$$
V_{3 \mathrm{~d}}=V / T, \quad \rho_{3 \mathrm{~d}}=\rho / T .
$$

The new variables (36) have the momentum dimensions of the 3-dimensional potential and field-squared. Moreover, (35) takes the form and the dimensional properties of a $(3+0)$-dimensional flow for the potential.

With the same line of arguments it can be easily seen that a finite volume setup does not support a nonvanishing condensate, effectively reducing to quantum mechanics for a large correlation length: if the cutoff scale $k$ is far smaller than the inverse size of the system, $k \ll 1 / L$, only the spatial zero mode contributes to the flow, effectively reducing it to a $0+1$-dimensional flow with only the frequency integral to be performed. This mimics a quantum mechanical flows, to wit

$$
\dot{V}_{k \ll 1 / L}(\rho) \rightarrow \frac{1}{2 L^{3}} \int \frac{\mathrm{d} q_{0}}{2 \pi} \frac{\dot{R}_{k}\left(q_{0},|\vec{q}|=0\right)}{q_{0}^{2}+R_{k}\left(q_{0},|\vec{q}|=0\right)+m_{k}^{2}(\rho)} .
$$

As in 35 the prefactor $1 / L^{3}$ can be absorbed with a similar rescaling of $V_{k}$ and $\rho$ similarly to (36), with

$$
V_{1 \mathrm{~d}}=V L^{3}, \quad \rho_{1 \mathrm{~d}}=\rho L^{3} .
$$

This renders (37) in a form that is identical to that of the quantum-mechanical $(0+1)$-dimensional flow for the potential. The latter flow does not admit a non-trivial minimum for the potential: there is no spontaneous symmetry breaking in quantum mechanics.

Finally, at finite temperature and for $k \ll \min (1 / L, T)$, the system reduces to the zero mode,

$$
\dot{V}_{k \ll \min (1 / L, T)}(\rho) \rightarrow \frac{T}{L^{3}} \frac{k^{2}}{k^{2}+m_{k}^{2}(\rho)},
$$

where, without loss of generality, we have used that $R_{k}(0)=k^{2}$ and $\dot{R}_{k}(0)=2 k^{2}$. Again this can be mapped into the standard flow by a rescaling of $V_{k}$ and $\rho$ similarly to $(36),(38)$ with

$$
V_{0 \mathrm{~d}}=V L^{3} / T, \quad \rho_{0 \mathrm{~d}}=\rho L^{3} / T,
$$

i.e. effectively taking $T / L^{3} \rightarrow 1$. This is the flow of a $(0+0)$-dimensional field theory, the generating functional being the one dimensional integral

$$
Z_{0 \mathrm{~d}, k}(J)=\int d \phi \exp \left\{-V_{\mathrm{od}, \Lambda}(\rho)-k^{2} \rho_{\text {od }}+J \phi_{\text {od }}\right\},
$$

where $\Lambda \ll \min (1 / L, T)$. This trivial flow has been studied in e.g. Ref. [29]: it does not admit a non-trivial minimum for the respective effective action $\Gamma_{\text {od }}[\phi]$.

\section{Condensates}

In a finite volume no long range spatial fluctuations are permitted. However, the latter ones are required for phases with spontaneous symmetry breaking and nonvanishing condensates. Only in one or more spatial directions and with infinite extent (at vanishing temperature) non-vanishing condensates survive for the present Ising universality class model $(O(1)$-symmetry).

The disappearance of condensates in a finite volume is easily seen in $O(N)$-models for $N>1$ due to the presence of massless Goldstone modes with mass functions $m_{k, \theta}^{2}(\rho)=V_{k}^{\prime}(\rho)$, where $\vec{\theta}$ are the $N-1$ Goldstone modes. Here, emphasising the differences to the Ising-type case $N=1$, we briefly discuss this case.

The flow of the minimum can be derived from the $t$ derivative of the equation of motion, $V_{k}^{\prime}\left(\rho_{0}\right)=0$ for $\rho_{0}>$ 0 . Its flow reads

$$
\partial_{t} V_{k}^{\prime}\left(\rho_{0}\right)=\dot{V}^{\prime}\left(\rho_{0}\right)+\dot{\rho}_{0} V^{\prime \prime}\left(\rho_{0}\right)=0,
$$

where the flow $\dot{V}_{k}^{\prime}$ is given in 16 . The masses of the Goldstone modes vanish in the broken phase, i.e. at a non-trivial minimum $\rho_{0}>0$. By resolving (42) for $\dot{\rho}_{0}$ we 
arrive at

$$
\begin{aligned}
\dot{\rho}_{0} & =-\frac{\dot{V}^{\prime}\left(\rho_{0}\right)}{V^{\prime \prime}\left(\rho_{0}\right)} \\
& =\frac{1}{2} \int_{q} \dot{R}_{k}(q)\left[\left(3+\rho_{0} \frac{\lambda_{3}}{\lambda_{2}}\right) G_{k, \rho}^{2}(q)+(N-1) G_{k, \theta}^{2}(q)\right] \\
& \rightarrow \frac{1}{k^{2}} \frac{T}{L^{3}}\left[\frac{3+\rho_{0} \frac{\lambda_{3}}{\lambda_{2}}}{\left(1+\frac{2 \rho_{0} \lambda_{2}}{k^{2}}\right)^{2}}+(N-1)\right] .
\end{aligned}
$$

The first equality in (43) provides the flow of $\rho_{0}$ in the local potential approximation in an $O(N)$-model. The first term in the second line is the radial contribution while the second term comes from the Goldstone modes with the massless propagator $G_{\theta}$. In $d \leq 2$ (infinite range) dimensions and $N>1$ the latter term does not tend to zero in the limit $k \rightarrow 0$ while the first term does with powers of $k^{2} / m_{k}^{2}$. The Goldstone term is positive, and hence the condensate is driven to zero with $k \rightarrow 0$, that is $t \rightarrow-\infty$. This reflects the Mermin-Wagner theorem.

In a finite volume and at finite temperature the flow reduces to the third line for $k \ll \min (T, 1 / L)$. Again, the singularity in the flow is apparent. As a consequence, $\rho_{0}$ is driven to zero with $k \rightarrow 0$. This is also seen in Yukawatype models such as the linear quark-meson models and atom-condensate models for ultracold atoms, see e.g. [15.

Note that this argument does not apply to $N=1$ with the discrete $Z_{2}$-symmetry. The $O(1)$ model (Ising universality class) has no Goldstone mode and the vanishing of the condensate for $d<2$ dimensions cannot be read-off directly from the flow of the condensate, 43 . In particular, the flow in 43 reduces at finite temperature and volume to

$$
\dot{\rho}_{0} \rightarrow k^{2} \frac{T}{L^{3}} \frac{3+\rho_{0} \frac{\lambda_{3}}{\lambda_{2}}}{\left(k^{2}+2 \rho_{0} \lambda_{2}\right)^{2}},
$$

for $k \ll \min (T, 1 / L)$. This relates to the fact that the Ising universality class, $N=1$, shows a phase transition in $d=2$. Below two dimensions, $d<2$, it does not show a phase transition.

We remark that this structure is not seen in a Taylor expansion about the flowing minimum $\rho_{0}$. For example, in the $\phi^{4}$-approximation the condensate does not necessarily vanish at vanishing cutoff scale: for a finite mass $m_{k}^{2}$ the flow of all couplings, including $\rho_{0}$ and the free energy $F_{k}$, can vanish with powers of $k^{2} / m_{k}^{2}$. Indeed, the non-vanishing minimum stays at higher orders at this expansion too. This hints at a failure of the polynomial expansion about $\rho_{0}$. Note, however, that an expansion about $\rho=0$ works in dimensions $d \leq 2$, and converges rather rapidly with the full solution of the flow equation for the potential. This suggests an expansion about the flowing minimum $\rho_{0}$ for $k \gtrsim 1 / L$ and one about $\rho=0$ for $k \ll 1 / L$.
Still, the vanishing of the condensate at finite volume and temperature is easily seen. For the sake of completeness we briefly present the related argument. Assume for the moment that $m_{k=0}^{2}\left(\rho_{0}\right)>0$. Then, for $k>0$ the potential has a non-convex regime with $m_{k}^{2}\left(\rho<\rho_{w}\right)<0$ and the turning point $\rho_{w}$ with $m_{k}^{2}\left(\rho_{w}\right)=0$. For this regime the relative strength of the free energy $\Delta V_{k}=V_{k}(\rho=$ $0)-F_{k}$ is governed by

$$
\Delta \dot{V}_{k}=\frac{1}{2} \int_{q} \dot{R}_{k}(q)\left(G_{k}(\rho, q)-G_{k}(q)\right),
$$

where $\rho \leq \rho_{w}$ is a $k$-independent field value in the nonconvex regime with $m_{k}^{2}\left(\rho<\rho_{w}\right)<0$, potentially leading to large flows close to the singularity triggered by $k^{2}+$ $m_{k}^{2}(\rho)=0$. For the current purpose it is sufficient to use the estimate

$$
\frac{1}{p^{2}+R_{k}\left(p^{2}\right)}<\frac{1}{p^{2}+R_{k}\left(p^{2}\right)+m_{k}^{2}\left(\rho<\rho_{w}\right)}
$$

and, hence, we estimate

$$
\begin{aligned}
\Delta \dot{V}_{k} & >\frac{1}{2} \int_{q} \dot{R}_{k}(q)\left(\frac{1}{p^{2}+R_{k}}-\frac{1}{p^{2}+R_{k}+m_{k}^{2}}\right) \\
& =\frac{1}{2} \int_{q} \dot{R}_{k}(q)\left(\frac{1}{p^{2}+R_{k}} m_{k}^{2} \frac{1}{p^{2}+R_{k}+m_{k}^{2}}\right) .
\end{aligned}
$$

In the limit $k /[\min (1 / L, T)] \rightarrow 0$ we arrive at

$$
\Delta \dot{V}_{k} \rightarrow \frac{T}{L^{3}}
$$

Evidently, the flow of $\Delta V_{k}$ does not vanish for $k \rightarrow 0$ and hence the difference turns negative: the minimum is at $\rho=0$. Note that an iteration of this argument also excludes the existence of several minima and, therefore, guarantees a smooth vanishing of the minimum $\rho_{0}$ with $k \rightarrow 0$.

For vanishing temperature or more generally infinite range dimensions $d<2$ the above argument can be generalised by discussion of the flow of the turning point $\rho_{w}$ of the radial mass function $m_{k}^{2}(\rho)$ with $m_{k}^{2}\left(\rho_{w}\right)=0$. We have $\rho_{w} \leq \rho_{0}$ for the $O(1)$-model and $\rho_{w}=\rho_{0}$ in the $O(N>1)$-model. The full analysis is beyond the scope of the present work and hence discussed elsewhere.

\section{THERMODYNAMICS IN A FINITE VOLUME}

One of the main goals of this work is the study of finite volume effects in the pressure,

$$
p=-\partial F / \partial \mathcal{V},
$$

with the free energy $F$ and the spatial volume $\mathcal{V}=L^{3}$. The free energy $F$ relates to the effective action $\Gamma\left[\phi_{\text {EoM }}\right]$ 


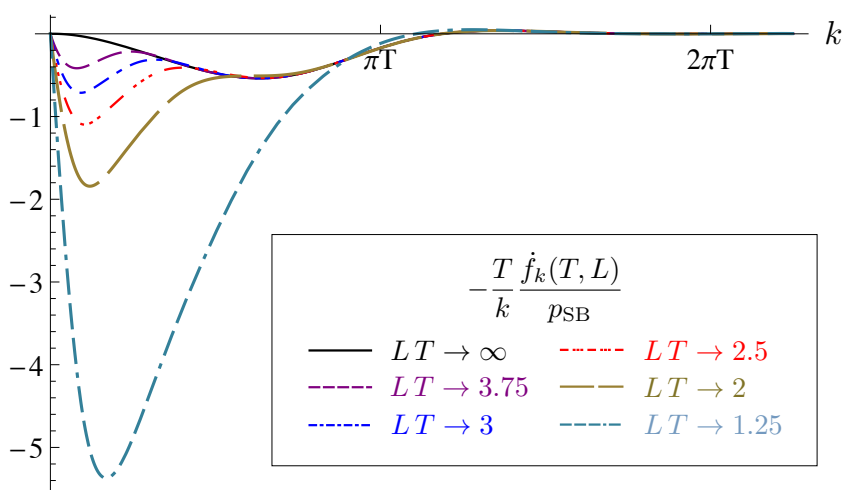

FIG. 2: Spatial volume dependence of the flow of the free energy density $f_{k}$ for a coupling $\lambda_{\Lambda}=0.5$ and an exponential regulator with $m=1$, cf. eq. 44.

on the solution of the EoM, $\phi_{\text {EoM }}$. It can be normalised (shifted) such that it vanishes in the combined infinite volume and zero temperature limit, that is

$$
F=T\left(\Gamma^{T, L}\left[\phi_{\text {EoM }}\right]-\Gamma^{0, \infty}\left[\phi_{\text {EoM }}\right]\right) .
$$

Eq. (50) is the free energy related to thermal and finite volume fluctuations, and the pressure $(49)$ with $(50)$ is the combined finite volume and thermal pressure. In the following we use the free energy density $f_{k}$ already introduced in 21] in the cutoff scale-dependent case. We have

$$
f_{k}=\frac{F_{k}}{\mathcal{V}}=\frac{\Gamma_{k}\left[\phi_{\text {EoM }}\right]}{\beta \mathcal{V}}, \quad \text { with } \quad \beta=\frac{1}{T}
$$

by extracting the trivial spatial volume factor in the static free energy. Eq. (50) and (51) entail that the pressure $p_{k}$ comprises the fluctuations induced from finite temperature and volume. The difference of free energies densities $f_{k}$ at finite and infinite volume relates to the Casimir force, for a FRG computation see Ref. [30].

The flow of the pressure is then given by the one of the free energy density and its volume or, equivalently, length derivative,

$$
\dot{p}_{k}(T)=-\dot{f}_{k}(T, L)-\frac{L}{3} \frac{\partial \dot{f}_{k}(T, L)}{\partial L},
$$

where we have used that $\mathcal{V}=L^{3}$. The flow of the free energy density $f_{k}$ is given by

$$
\dot{f}_{k}(T, L)=\frac{1}{2} \sum_{q} G_{k}^{T, L}(q) \dot{R}_{k}(q)-\frac{1}{2} \int_{q} G_{k}^{0, \infty}(q) \dot{R}_{k}(q),
$$

where the sign $\sum_{q}$ stands for a sum over frequencies and discrete spatial momenta. In Fig. 2 the flow of the free energy density, (53), is shown for different volumina.

In 53 the first term is the finite temperature flow

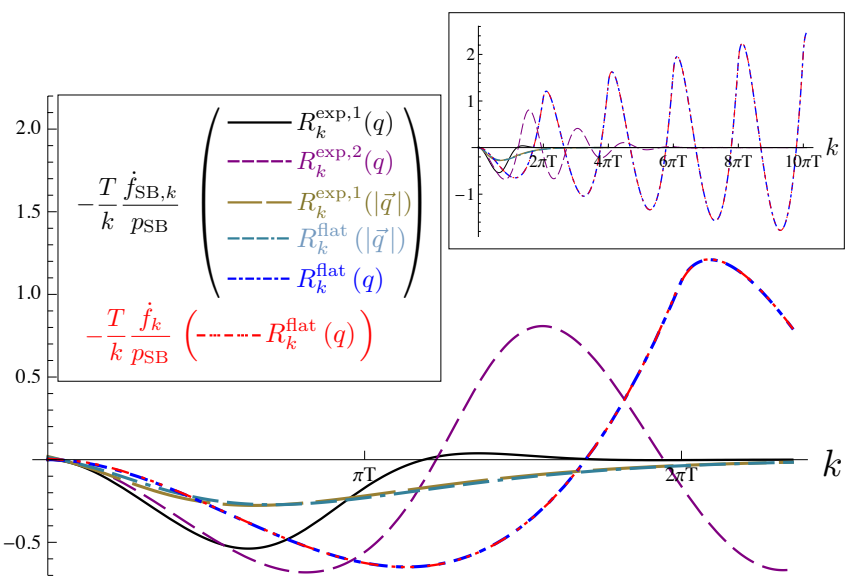

FIG. 3: Flow of the Stefan-Boltzmann pressure $p_{\mathrm{SB}, k}=$ $-f_{\mathrm{SB}, k}(T, \infty)$ for different regulator, and the full pressure for $R_{k}^{\text {flat }}$, in an infinite volume, with $\lambda_{\Lambda}=0.5$ for the full result.

involving the thermal and/or finite volume propagator $G_{k}^{T, L}(q)$, whereas the second term is the normalisation in terms of the vacuum propagator $G_{k}^{T=0, L=\infty}(q)$. Moreover, in the present LPA approximation the flow of the free energy density $\dot{f}_{k}$ at a given cutoff scale $k$ is simply given by the difference of the flows of the effective potential,

$$
\dot{f}_{k}=\dot{V}_{k}^{T, L}\left(\rho_{0}\right)-\dot{V}_{k}^{0, \infty},
$$

cf. section II. The flow equation for the infinite volume pressure is given by that of the free energy density,

$$
\dot{p}_{k}(T)=-\lim _{L \rightarrow \infty} \dot{f}_{k}(T, L),
$$

as the $L$-derivative of $\dot{f}_{k}$ vanishes faster than $1 / L$. The flow of the thermal pressure $\dot{p}_{k},(55)$, is shown for different regulators in Fig. 3 .

The influence of the second term in a finite volume can readily be discussed qualitatively. It is already wellknown from purely thermal flows that $\dot{f}_{k}>0$ for $k \lesssim$ $\min (T, 1 / L)$. It is this cutoff regime where the main contribution to the pressure is collected during the flow, leading to a positive pressure, see also Fig. 2. For larger cutoff scales the flow switched sign, $\dot{f}_{k} \lesssim 0$ for $k \lesssim \min (T, 1 / L)$. As the volume and temperature pressure grows with decreasing volume and/or increasing temperature the above properties imply

$$
\begin{aligned}
& \frac{\partial \dot{f}_{k}}{\partial L}<0 \quad \text { for } \quad k \lesssim \min (T, 1 / L), \\
& \frac{\partial \dot{f}_{k}}{\partial L} \gtrsim 0 \quad \text { for } \quad k \gtrsim \min (T, 1 / L) .
\end{aligned}
$$

In summary the integrated contributions from $-1 / 3 L \partial_{L} \dot{f}_{k}$ decrease the pressure in a finite volume, and the free energy density $f_{k=0}$ gives an upper 
estimate for the pressure.

\section{A. Stefan-Boltzmann pressure for three-dimensional flat regulator}

A simple but instructive example for the properties and definitions discussed above is the tree-level free energy density $f_{\mathrm{SB}, k}$ and pressure $p_{\mathrm{SB}, k}$, i.e. the StefanBoltzmann contributions, for two reasons. Firstly, it illustrates on an analytic level the computations and results presented in the next Section V]. Secondly, and more importantly, it demonstrates constraints on the regulators emerging from finite temperature or volume in a clear way.

First, we compute $p_{\mathrm{SB}}$ in the infinite volume limit with the three-dimensional flat regulator, see (5). This regulator is often used as analytic computations can be pushed far. The free propagator in the presence of $R_{k}^{\text {flat }}(|\vec{q}|)$ is simply given by

$$
G_{k}^{(0)}(q)=\frac{1}{q_{0}^{2}+k^{2}} \theta\left(k^{2}-\vec{q}^{2}\right)+\frac{1}{q_{0}^{2}+\vec{q}^{2}} \theta\left(\vec{q}^{2}-k^{2}\right) .
$$

With the propagator in 57) and $\dot{R}_{k}^{\text {flat }}=2 k^{2} \theta\left(k^{2}-\vec{q}^{2}\right)$, the flow for the infinite volume Stefan-Boltzmann pressure 55) can be given analytically, see e.g. 31, 32,

$$
\begin{aligned}
\dot{p}_{\mathrm{SB}, k}(T) & =-\int_{0}^{k} \frac{\mathrm{d} q_{s}}{2 \pi^{2}}\left(T \sum_{n=-\infty}^{\infty} \frac{k^{2} q_{s}^{2}}{k^{2}+\omega_{n}^{2}}-\int_{q_{0}} \frac{k^{2} q_{s}^{2}}{k^{2}+q_{0}^{2}}\right) \\
& =-\frac{k^{4}}{12 \pi^{2}}\left(\operatorname{coth} \frac{k}{2 T}-1\right)
\end{aligned}
$$

For large RG scales compared to the temperature this turns into

$$
\stackrel{k / T \rightarrow \infty}{\longrightarrow}-\frac{k^{4}}{6 \pi^{2}} \exp \left(-\frac{k}{T}\right),
$$

where $q_{s}=\sqrt{\vec{q}^{2}}$ and $\int_{q_{0}}=\int_{-\infty}^{\infty} \frac{\mathrm{d} q_{0}}{2 \pi}$. The flow 58 and that for other regulators is shown in Fig. 3 . Eq. (58) entails that for large cutoff scales $k / T \rightarrow \infty$ the flow decays exponentially with $\exp (-k / T)$ in line with 30 . This is expected as the cutoff used in (58) does not affect the frequency sum, and reflects the fast decay of thermal fluctuations in the presence of large mass scales. Hence, the pressure at the initial scale $\Lambda \gg T$ can be safely put to zero, $p_{\mathrm{SB}, \Lambda}=0$. The integration over the RG scale $k$ from $\infty$ to 0 gives the correct pressure $p_{\mathrm{SB}}$ of a gas of free scalar particles,

$$
p_{\mathrm{SB}}(T)=\int_{\infty}^{0} \frac{\mathrm{d} k}{k} \dot{p}_{\mathrm{SB}, k}(T)=\frac{\pi^{2} T^{4}}{90} .
$$

The Stefan-Boltzmann expression 58 is easily upgraded to the full flow. In the latter case we simply have to substitute $k^{2} \rightarrow k^{2}+m_{k}^{2}$ in the denominators in the first line of 58 and arrive at

$$
\begin{aligned}
\dot{p}_{k}(T)= & -\frac{k^{4}}{12 \pi^{2}} \frac{1}{\sqrt{1+\frac{m_{k}^{T^{2}}}{k^{2}}}}\left(\operatorname{coth} \frac{k}{2 T} \sqrt{1+\frac{m_{k}^{T^{2}}}{2}}-1\right) \\
& -\frac{k^{4}}{12 \pi^{2}}\left(\frac{1}{\sqrt{1+\frac{m_{k}^{T^{2}}}{k^{2}}}}-\frac{1}{\sqrt{1+\frac{m_{k}^{0^{2}}}{k^{2}}}}\right)
\end{aligned}
$$

The term in the first line of 60 is decaying exponentially for large cutoff scales $k / T \rightarrow \infty$ also in the presence of non-vanishing mass function $m_{k}^{T}$. In turn, the term in the second line is proportional to $\left(m_{k}^{T}\right)^{2}-\left(m_{k}^{T=0}\right)^{2}$ : the exponential decay of the full flow in 60 hinges on the exponential decay of the difference of the mass functions at finite temperature and vanishing temperature. The flow in $(60)$ already encodes the one of differences of the effective potential with $m_{k} \rightarrow m_{k}(\rho)$. Evidently, the exponential decay for $\left(m_{k}^{T}(\rho)\right)^{2}-\left(m_{k}^{T=0}(\rho)\right)^{2}$ is encoded in the flow. This simple but instructive example already illustrates the main features of the thermal and finite volume properties under investigation: the flow reflects the thermal decay with large mass scales in case that this is also present for the flowing couplings.

We now repeat this computation with the threedimensional flat regulator in the general case with a finite volume and at finite temperature. For the sake of simplicity we first drop the $\partial_{L} f_{k}$-term in 52 . The threedimensional spatial integral turns into sums, and we arrive at the Stefan-Boltzmann expression for the flow of the free energy density,

$$
\begin{aligned}
\dot{f}_{\mathrm{SB}, k} & =\frac{k}{2 L^{3}} \sum_{\vec{q}^{2} \leq k^{2}} \operatorname{coth} \frac{k}{2 T}-\int_{q_{0}} \frac{k^{2} q_{s}^{2}}{k^{2}+q_{0}^{2}} \\
& =\frac{k}{2 L^{3}} \sum_{\vec{q}^{2} \leq k^{2}}\left(\operatorname{coth} \frac{k}{2 T}-1\right)-\left(\frac{k^{4}}{12 \pi^{2}}-\frac{k}{2 L^{3}} \sum_{\vec{q}^{2} \leq k^{2}}\right),
\end{aligned}
$$

in a splitting similar to that in 60. In 61 we have

$$
\vec{q}^{2}=\left(\frac{2 \pi}{L}\right)^{2} \vec{n}^{2}, \quad \text { with } \quad \vec{n}=\left(n_{1}, n_{2}, n_{3}\right) .
$$

Results for different regulators are shown in Fig. 4.

The first term in parenthesis in the second line of 61 decays exponentially with $\exp (-k / T)$ for large $k / T$, but does not decay exponentially with $k L$ for fixed $k / T$. This signals the failure of the three-dimensional flat regulator to reflect the exponential decay of finite volume fluctuations in the presence of large mass scales in a similar decay with the cutoff scale.

For a direct access to the finite volume decay we take the limit $T \rightarrow 0$. Then, the first term in parenthesis 


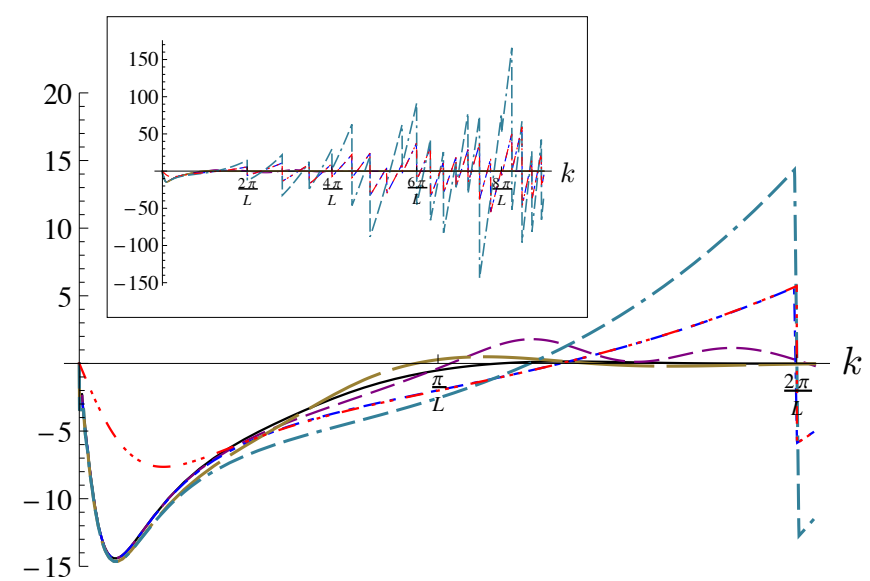

FIG. 4: Flow of the Stefan-Boltzmann free energy density $-f_{\mathrm{SB}, k}$ for different regulators, and and the full free energy density, $-f_{k}$, for $R_{k}^{\text {flat }}$, in an finite volume with relative lengths $L T=1$ and $\lambda_{\Lambda}=0.5$ for the full result. The colour coding and normalisation is the same as in Fig. 3 .

vanishes and we are left with the second term. This limit gives the flow of the free energy density in a finite volume normalised with that in an infinite volume. It is expected to decay exponentially with $k L \rightarrow \infty$ similar to a thermal flow. Instead, the combined flow fluctuates about zero with a rising enveloping amplitude proportional to $k^{2}$.

The sum in the second term simply counts the number of momentum points in a three-dimensional sphere with radius $k$. Within the numerical precision of the present work we find for a $d$-dimensional sphere,

$$
\sum_{q^{2} \leq k^{2}} \rightarrow \frac{\pi^{d / 2}}{\Gamma\left(\frac{d+2}{2}\right)}\left(\frac{k L}{2 \pi}\right)^{d}+O\left(k^{\theta_{d}}\right), \quad \theta_{d \geq 3}=d-2,
$$

where $q^{2}=(2 \pi)^{2} / L^{2}\left(n_{1}^{2}+\cdots+n_{d}^{2}\right)$ is the $d$-dimensional discrete momentum squared. For $d=1$ we have trivially $\theta_{1}=0$. Note that while the sub-leading behaviour in (63) has been proven for $d \geq 4$, it is still subject to ongoing research for $d=2,3$, with $d=2$ being the Gauß circle problem. The best estimates we found in the literature are $\theta_{3} \leq 21 / 16$ 33] and $1 / 2<\theta_{2} \leq 131 / 208$ [34. In Appendix A for illustration, we have performed a similar computation for a regulator that achieves a flat propagator in a spatial momentum box with box lengths $2 k$. Such a regulator allows for fully analytic computations, and is even more adapted to the symmetries of the spatial box.

With 63 the Stefan-Boltzmann flow $\dot{f}_{\mathrm{SB}, k}$ in a finite volume diverges proportional to $k^{2}$ for the threedimensional flat regulator. This asks for a non-vanishing initial condition $f_{\mathrm{SB}, \Lambda}$ at the initial cutoff scale $k=\Lambda$ in order to guarantee that $f_{\mathrm{SB}, k=0}$ is the tree level free energy density. Corrections of the thermodynamics due to

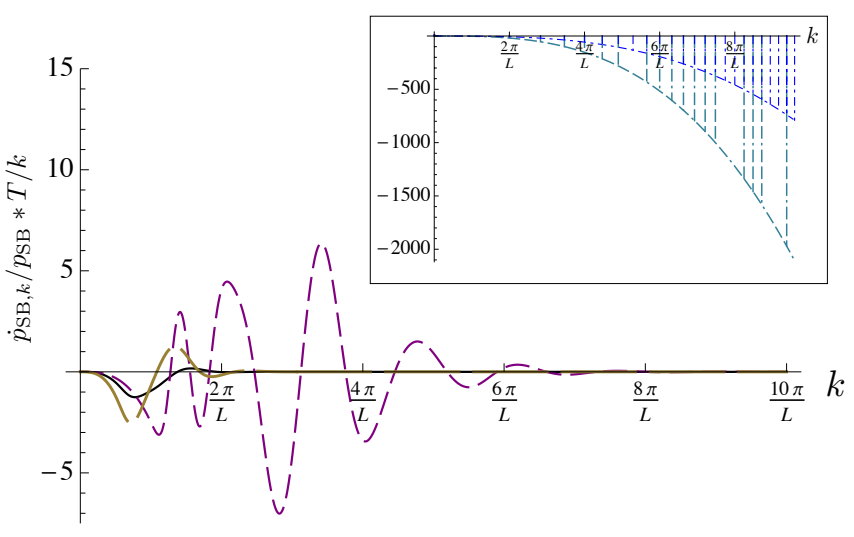

FIG. 5: Flow of the pressure $p_{\mathrm{SB}, k}$ for different regulators in a finite volume with relative lengths $L T=1$. The colour coding and normalisation is the same as in Fig. 3

the full dynamics are encoded in the fluctuating part

$$
\begin{aligned}
f_{k}-f_{\mathrm{SB}, k} \propto & -\frac{k^{4}}{12 \pi^{2}}\left(\frac{1}{\sqrt{1+\frac{m_{k}^{0, \infty^{2}}}{k^{2}}}}-\frac{1}{\sqrt{1+\frac{m_{k}^{T, L^{2}}}{k^{2}}}}\right) \\
+ & {\left[\frac{k}{2 L^{3}} \sum_{\vec{q}^{2} \leq k^{2}}-\frac{k^{4}}{12 \pi^{2}}\right]\left(\frac{1}{\sqrt{1+\frac{m_{k}^{T, L^{2}}}{k^{2}}}}-1\right), }
\end{aligned}
$$

where we have dropped the exponentially decaying parts. The term on the right hand side of the first line in $\sqrt{64}$ is proportional to $k^{2}\left(\left(m_{k}^{T, L}\right)^{2}-\left(m_{k}^{0, \infty}\right)^{2}\right)$. The mass difference tends to a constant, albeit small (up to logarithmic corrections), for large scales. In turn, the second line is proportional to the difference of three-dimensional integral and three-dimensional sum, that is the sub-leading term in 63. Hence, its total scaling is proportional to $k^{1+\theta_{3}} \approx k^{2}$.

The above analysis also entails that for the present regulator, $R_{k}^{\text {flat }}\left(\vec{q}^{2}\right)$ the second term in the flow of the pressure, (52), a priori cannot be dropped even in the infinite volume limit. It can be easily computed from (61), and reads

$$
-\frac{L}{3} \frac{\dot{f}_{\mathrm{SB}, k}}{\partial L}=\dot{f}_{\mathrm{SB}, k}+\frac{k^{4}}{12 \pi^{2}}-\frac{1}{3} \frac{k^{3}}{L^{3}} \operatorname{coth} \frac{k}{2 T} \sum_{\vec{n} \in \mathbb{Z}^{3}} \delta\left(k^{2}-\vec{q}^{2}\right),
$$

where the second term is vanishing everywhere except for $k^{2}=(2 \pi / L)^{2}\left(n_{1}^{2}+n_{2}^{3}+n_{3}^{3}\right)$, simply collecting the contributions at the discrete momentum values. Using both terms, (61) and (65), in the flow equation for the pres- 
sure, 52, we arrive at the simple expression

$$
\dot{p}_{\mathrm{SB}, k}=\frac{k^{4}}{12 \pi^{2}}-\frac{1}{3} \frac{k^{3}}{L^{3}} \operatorname{coth} \frac{k}{2 T} \sum_{\vec{n} \in \mathbb{Z}^{3}} \delta\left(k^{2}-\vec{q}^{2}\right) .
$$

The flow of the Stefan-Boltzmann pressure for the threedimensional flat regulator is given by a $k^{4}$-term and a $\delta$-function contribution that peaks at the discrete spatial momenta. For the full pressure flow we can repeat the same analysis as for the flow of the free energy density leading to similar $k^{2}$-corrections.

In Fig. 5 we show the flow of the free energy density for the three-dimensional flat regulator in comparison to their Stefan-Boltzmann counterparts. Again, the differences are negligible for large cutoff scales as $m_{k}^{2} / k^{2} \ll 1$. This limit is usually achieved in theories or models with small ultraviolet fluctuations in the validity range of the theories. Note that in the present $\phi^{4}$-theory the Landau pole is hit at a large cutoff $k_{\text {Landau }}$, and one has to safely stay below this scale. We also emphasise that both, the flow of the free energy density, $-\dot{f}_{k}$ in 61 , and that of the pressure, $\dot{p}_{k}$ in (66), tend towards (58) in the infinite volume limit. For $T \rightarrow 0$ and $L \rightarrow \infty$ both flows vanish.

The simple example of thermodynamical observables studied in the present Section has already taught us an important lesson: flows with non-analytic regulators do not reflect the physical exponential decay with large mass scales in the decay with the cutoff scale. While the latter property is not necessary in order to guarantee the former physical one, it is potentially cumbersome to achieve in approximations.

\section{B. Stefan-Boltzmann pressure for four-dimensional flat regulator}

For the four-dimensional flat regulator, (5), the treelevel propagator reads

$$
G_{k}^{(0)}(q)=\frac{1}{k^{2}} \theta\left(k^{2}-q^{2}\right)+\frac{1}{q^{2}} \theta\left(q^{2}-k^{2}\right),
$$

with the momentum squared $q^{2}$ only taking discrete values

$$
q^{2}=(2 \pi T)^{2} n_{0}^{2}+\left(\frac{2 \pi}{L}\right)^{2} \vec{n}^{2}, \quad n_{\mu} \in \mathbb{Z},
$$

and $\vec{n}=\left(n_{1}, n_{2}, n_{3}\right)$. We have chosen all lengths to be equal, $L_{i}=L$. With the propagator 67 and $\dot{R}_{k}^{\text {flat }}(q)=$ $2 k^{2} \theta\left(k^{2}-q^{2}\right)$, the flow of the free energy density is given by

$$
\dot{f}_{\mathrm{SB}, k}=\frac{T}{L^{3}} \sum_{q^{2} \leq k^{2}}-\int_{q^{2} \leq k^{2}} \frac{\mathrm{d}^{4} q}{(2 \pi)^{4}}=\frac{T}{L^{3}} \sum_{q^{2} \leq k^{2}}-\frac{k^{4}}{32 \pi^{2}} .
$$

Similarily to the computation above for the threedimensional flat regulator, we have to add the second

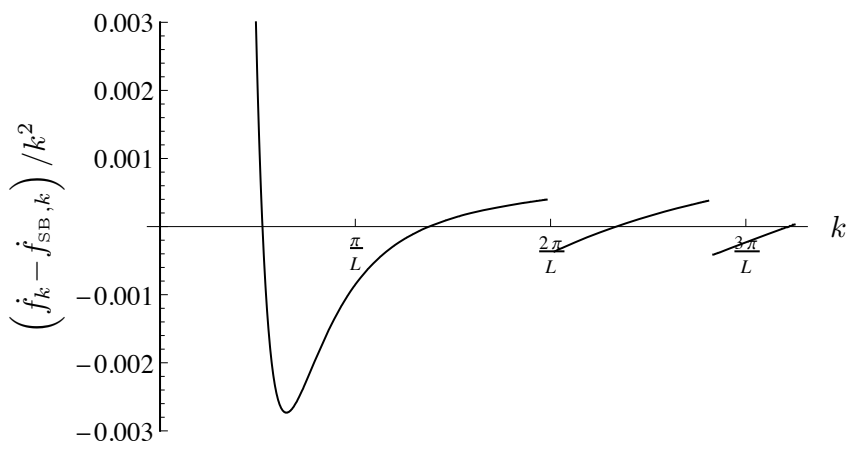

FIG. 6: The difference of the flow of the free energy density contribution for the four-dimensional flat regulator $R_{k}^{\text {flat }}(q)$.

term in 52. This leads to the simple flow

$$
\dot{p}_{\mathrm{SB}, k}=\frac{k^{4}}{32 \pi^{2}}-\frac{2}{3} \frac{T}{L^{3}} \sum_{n_{\mu} \in \mathbb{Z}} \vec{q}^{2} \delta\left(k^{2}-q^{2}\right),
$$

see Fig. 5. The spatial and four-dimensional momenta squared $\vec{q}^{2}$ and $q^{2}$ are defined in 62 and $(68)$, respectively.

Similar to the case of the three-dimensional flat regulator we conclude that the flow of the free action density is sensitive to finite volume effects for all cutoff scales for the four-dimensional flat regulator. With a similar analysis as in the last Section IV A it follows that there are subleading terms proportional to $k^{2}\left(\left(m_{k}^{T, L}\right)^{2}-\left(m_{k}^{0, \infty}\right)^{2}\right)$ and $k^{2}\left(m_{k}^{T, L}\right)^{2}$. For the choice $m_{k}^{2} / k^{2} \ll 1$, being the initial value in both finite and infinite volume as well as zero and non-zero temperature, at large cutoff scales the corrections are small. In Fig. 4 we show the flow of the free energy density for the four-dimensional flat regulator in comparison to their Stefan-Boltzmann counterparts, as well as the Stefan-Boltzmann flows for other regulators. In Fig. 6 we show the difference of the flow of the free energy density. This difference clearly shows the ultraviolet sensitivity of the flow with respect to finite volume effects.

In the infinite volume limit both, $(69)$ and $(70)$ tend towards the infinite volume flow. For $T \rightarrow 0$ and $L \rightarrow \infty$ both flows vanish. We also conclude that for both, the three-dimensional and four-dimensional flat regulators, the thermodynamics is approximately accessible for $m_{\Lambda}^{2} / \Lambda^{2} \ll 1$ at the expense of a trivial non-vanishing initial condition $f_{\Lambda}=f_{\mathrm{SB}, \Lambda}$ and $p_{\Lambda}=p_{\mathrm{SB}, \Lambda}$. However, since the flow of $f_{k}$ and $p_{k}$ only depends on the couplings, the Stefan-Boltzmann terms and the $\Delta \dot{f}_{k}, \Delta \dot{p}_{k}$ can be integrated separately: the total free energy density/pressure is simply given by

$$
p_{k=0} \approx p_{\mathrm{SB}}(T, L)+\int_{\Lambda}^{0} \frac{d k}{k} \Delta \dot{p}_{k}
$$

with the trivial Stefan-Boltzmann pressure $p_{\mathrm{SB}}(T, L)$ in 
a finite volume and the integrated flow of $\Delta \dot{p}_{k}$.

The findings of the present and the last section carry over to the theory in the presence of three- and fourdimensional sharp regulators, (6). Indeed, in these cases the ultraviolet growth is very similar. Below (6) it has been discussed that the theory in the presence of sharp regulators is directly related to respective ultraviolet momentum cutoff regularisations of DSEs in a finite volume and at finite temperature, 11 14. Consequently the respective correlation functions miss the exponential decays unless one applies a volume and/or temperature dependent renormalisation procedure. Note that only for the present thermodynamical observables, pressure and free energy density, this shows up as a growth with the ultraviolet cutoff scale $\Lambda$, in higher order correlation functions the missing exponential decay is hidden in polynomially decaying terms.

We add that these findings apply to all situations with a sharp momentum cut-off for discrete momentum modes. This includes in particular Landau level sums in the presence of magnetic fields. There it is wellknown that the critical temperature, and therefore the phenomenon of (inverse) magnetic catalysis shows an exponential sensitivity with respect to the scales of the problem. Hence it is particularly sensitive to the presence or absence of the exponential decay discussed above. For FRG-applications to the (inverse) magnetic catalysis see 35-39, for other approaches with ultraviolet momentum cutoffs including DSEs and perturbation theory, see the recent reviews [40, 41].

We close with the remark that it is not simply the missing differentiability of the regulator that spoils the decay with the cutoff scale. It originates in the non-analytic behaviour of the regulator at $p^{2}=k^{2}$. In Appendix B we test the smooth modifications of the flat regulator suggested in 42 that are $\mathcal{C}^{\infty}$ at $p^{2}=k^{2}$ at the expense of an essential singularity $\propto \exp \left\{-\right.$ const./ $\left.\left(1-p^{2} / k^{2}\right)\right\}$ for spatial momentum approaching $k$ from below, $p^{2} \rightarrow k_{-}^{2}$. They show the same missing exponential decay in the flow, although the smoothening of the non-analyticity leads to smaller fluctuations at large cutoff scales, see Fig. 20 in Appendix B. This is not surprising as it is well-known from the thermal case that the exponential decay reflects the pole structure of the loop integrals in the complex plane if the Matsubara sum is rewritten as the (original) contour integral. This suggests that analytic versions of the flat regulator have all the necessary properties for optimising flows at finite volume and temperature in the sense of the optimisation criterion in [18].

\section{Pressure for general regulators}

The flow of the pressure for general regulators is based on that of the free energy density and its length derivative. More generally the length-derivative of the flow of the effective action is given by

$$
\begin{aligned}
L \frac{\partial \dot{\Gamma}_{k}}{\partial L}= & \oiint_{q} \vec{q}^{2} \partial_{\vec{q}^{2}}\left[G_{k} \dot{R}_{k}(q)\right] \\
& -\left.\frac{1}{2} \oiint_{q} G_{k} \dot{R}_{k} G_{k}(q) L \frac{\partial \Gamma_{k}^{(2)}(q)}{\partial L}\right|_{\vec{q}^{2}},
\end{aligned}
$$

where the last term hits the $L$-dependences of the vertices. Eq. 72 can be inserted in the definition of $f_{k}$, (51), to obtain the $L$-derivative of the general flow of the free energy density. In the present LPA-approximation the second term in the flow of the pressure, 52 , reduces to

$$
-\frac{L}{3} \frac{\partial \dot{f}_{k}}{\partial L}=\dot{f}_{k}+\dot{V}_{k}^{0, \infty}+\frac{1}{6} \oiint_{q}\left(2 \vec{q}^{2} \partial_{\vec{q}^{2}}-L \frac{\partial m_{k}^{2}}{\partial L} \partial_{m_{k}^{2}}\right) G_{k} \dot{R}_{k},
$$

see Fig. 5. This leaves us with the task of computing the $L$-dependence of the couplings. This is either done by performing computations at $L$ and $L+\epsilon$ and taking discrete derivatives or by using flow equations for the $L$ derivatives in a fixed volume, see Appendix C.

We close the present Section with a brief summary of the results. In the Figs. 3, 4, 5 the findings of this Section are illustrated. In Fig. 3 the Stefan-Boltzmann pressure in an infinite volume is shown for different regulators and the full pressure for the four-dimensional flat regulator. One sees both the exponential decay with $k$ for analytic regulators (in the frequency $p_{0}$ ) and the power law rise for non-analytic regulators (in the frequency). All flows are apparently smooth in contradistinction to the finite volume flows depicted in Figs. 4. 5. This is related to the fact that the chosen regulators with a $q_{0^{-}}$ dependence are functions of $q_{0}^{2}+\vec{q}^{2}$. This entails that the jumps for non-analytic regulators, that are related to the discrete Matsubara frequencies are smoothened out by the spatial momentum integration. Regulators of the form $R_{k}\left(q_{0}, \vec{q}\right)=R_{0, k}\left(q_{0}^{2}\right)+R_{s, k}\left(\vec{q}^{2}\right)$ with non-analytic regulators $R_{0, k}$ lead to jumps similarly to that shown in Fig. 4.

In Fig. 5 the Stefan-Boltzmann pressure in a finite volume is shown for different regulators. Again one sees both the exponential decay with $k$ for analytic regulators and the polynomial rise for non-analytic regulators. Additionally, one has discontinuities as described in (61), (69) for the flow of the free energy density and in 65 , 770 for the flow of the pressure. The polynomial rise of the free energy density and the pressure with $k^{3}$ and $k^{4}$ in a finite volume for the three-dimensional and fourdimensional flat regulator, respectively, hint at similar, but reduced problems for the flow of the mass $m_{k}^{2}$ (or $\left.\kappa_{k}\right)$ in both cases. The $k$-power counting of these flows has an additional factor $k^{-2}$ naively leading to rising volume-dependence with $k$ and $k^{2}$, respectively. If the naive power counting holds, some care is required with the initial conditions for the mass, as then it varies with 


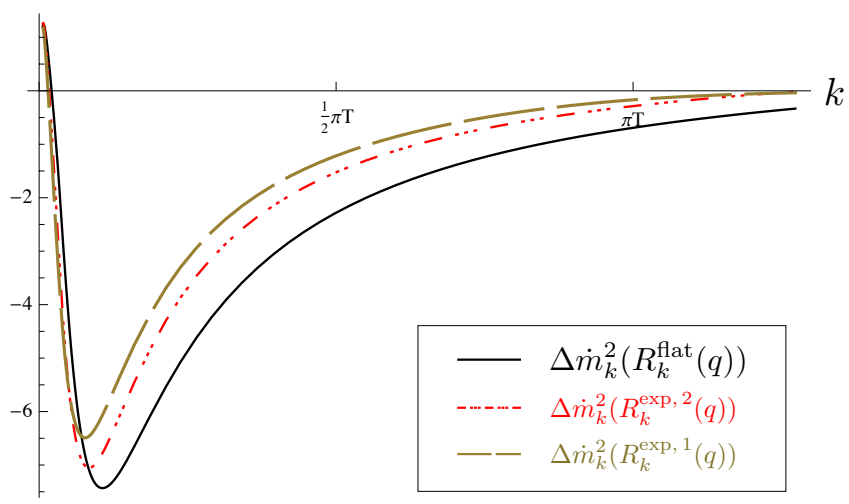

FIG. 7: Normalised mass difference $\Delta m_{k}^{2}$ at finite temperature $T=0.25$ in an infinite volume as defined in 76 .

the volume and the temperature.

For the coupling constant $\lambda_{k}$ we expect a $1 / k$ suppression of the flow for the three-dimensional flat regulator and a $k^{0}$-dependence for the four-dimensional flat regulator. This translates into a slow convergence of the flow towards the infinite volume flow in the first case, hence asking for a very large initial cutoff scale. In the latter case this leads to a logarithmic dependence with $k$ of the initial condition on the volume and temperature, and requires some care.

\section{COUPLINGS IN A FINITE VOLUME}

We have already discussed in the introduction and in more details in Section III, that a quantitative grip on the thermal, volume and cutoff scale dependence is pivotal for getting hold of in particular thermodynamical quantities and the equation of state. Moreover, the analytic results in the last Section [V] indicate the problems of non-analytic regulator choices such as the flat regulator and the sharp cutoff.

\section{A. Thermal \& finite volume decay}

In the following, we discuss the quantitative behaviour of the flows of couplings and thermodynamical observables in dependence of the choice of regulators. Despite the choice of regulator both finite temperature and volume are pure infrared effects, their effects are damped above energy scales which are typically of the order of the first frequency either in temporal or spatial direction. In other words, for scales much larger than the volume or temperature the theory turns into the infinite volume vacuum theory. The details of the decay are physically important, for the decay behaviour in mass scales and momentum scales see (30), (32) and (33) for the important example of the Tan-contact.

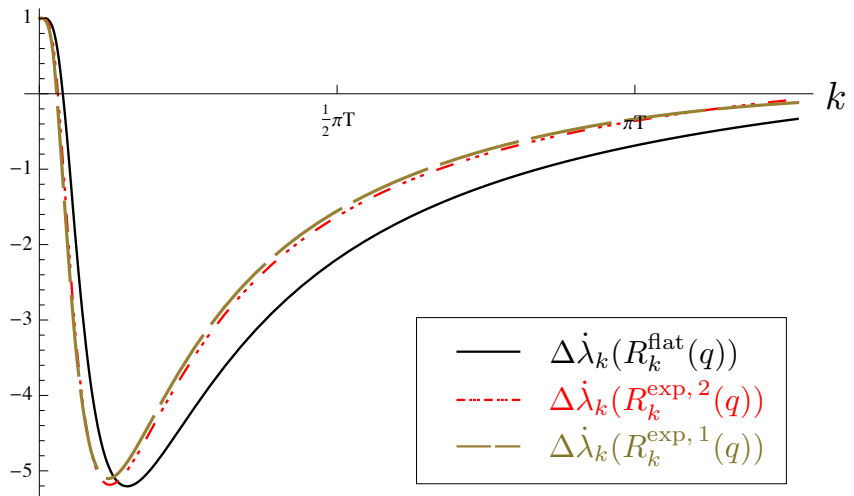

FIG. 8: Normalised $\phi^{4}$-coupling difference $\Delta \lambda$ at finite temperature $T=0.25$ in an infinite volume as defined in $(76)$.

In methods where fluctuations at all scales are integrated out at once this usually happens around the typical frequency or spatial momentum scale given by the first frequency $2 \pi T$ of $2 \pi / L$. This feature does not necessarily hold for the momentum cutoff scale $k$ flow. As has been already seen in Section IV for thermodynamical observables, this cutoff scale depends strongly on the shape of the regulator. More precisely, it depends on the way the infrared modes are suppressed: the sharper this suppression is implemented, the further in $k$ one has to go to lose sensitivity to thermal or finite volume effects. In particular for non-analytic regulators such as the flat regulator and the sharp cutoff this loss of sensitivity is delayed to infinity.

For the regulator shapes given in (4), (5) and (6) regulating either four-dimensionally or in spatial directions only, the thermal flow of the tree-level pressure $\dot{p}_{\mathrm{SB}, k}$ is given in Fig. 3. In an infinite volume the problem of a "mode-counting" cutoff can be circumvented by regulating the spatial momenta only. In contrast, in a finite volume this generic property rules out sharp and flat regulators for practical computations. We note that this is not cured by the inclusion of running masses and couplings, as also seen in Fig. 4 anticipating results below.

We conclude that the exponential thermal decay in (30) does not simply hold with $m_{\text {gap }} \rightarrow k$, even though this has been observed for the three-dimensional flat regulator, see (58). In general, one has to allow for a prefactor $c_{T}$ with

$$
\exp \left(-\frac{m_{\text {gap }}}{T}\right) \rightarrow \exp \left(-c_{T} \frac{k}{T}\right), \quad c_{T} \in[0,1] .
$$

For regulators that only depend on spatial momenta and have an (effective) infrared mass $R_{k}(0)=k^{2}$ the upper limit, $c_{T}=1$, is saturated: the sum over the Matsubara modes is not cut-off, and the thermal behaviour is untouched. With the dominant mass scale $k$ for $k \rightarrow \infty$ this leads to the standard thermal suppression with $c_{T}=1$. Note that strictly speaking the upper limit is not well 
defined as it depends on the mass scale introduced with the regulator. Only if the latter scale is identical with $k$ the prefactor $c_{T}$ satisfies (74).

In turn, for regulators that are non-analytic in frequency space such as the four-dimensional flat regulator, the exponential suppression is lost and the lower bound is saturated, $c_{T}=0$.

In a finite volume the decay towards the vacuum limit is induced by both, thermal and volume fluctuations. Hence, it leads to a generalisation of the thermal decay (30), (74). For (53), we parametrise the decay in the limit

$$
\lim _{k / T, k L \rightarrow \infty}\left|\Delta \mathcal{O}_{k}^{T, L}\right| \propto A\left(\frac{k}{T}, k L\right) \exp \left(-c_{T}(L T) \frac{k}{T}\right),
$$

where the function $A$ now encodes thermal and volume effects, and

$$
\Delta \mathcal{O}_{k}^{T, L} \equiv \frac{\mathcal{O}_{k}^{T, L}-\mathcal{O}_{k}^{0, \infty}}{\mathcal{O}_{k}^{0, \infty}} .
$$

$\Delta \mathcal{O}_{k}^{T, L}$ is the difference of an observable $\mathcal{O}_{k}^{T, L}$ at finite volume and temperature and infinite volume and vanishing temperature, normalised by its flow at $T=1 / L=0$. In Fig. 7 and Fig. 8 the normalised difference $\Delta \mathcal{O}_{k}$ is shown for the mass and the coupling difference respectively for finite temperature in an infinite volume.

The definition (76) also applies to flows or diagrammatic kernels of flows, the threshold functions. Flow equations of thermodynamical observables and vertices depend on the cutoff derivative of the regulator and powers of propagators. The decay of general flows can hence be measured by that of

$$
\mathcal{O}_{k, n}^{T, L}=\int_{q} G_{k}(q)^{n} \dot{R}_{k}(q) .
$$

In Fig. 9 we show the decay of general flows for different regulators, precisely, we show

$$
\frac{\mathcal{O}_{k, n}^{T, L}-\mathcal{O}_{k, n}^{0, \infty}}{k^{d-2(n-1)}},
$$

with the definition $(77)$ for $n=1,2,3,4$. We normalise with respect to the canonical dimension $k^{d-2(n-1)}$. In the presence of an exponential decay, that is $c_{T} \neq 0$ for $\Delta \mathcal{O}_{k, 1}^{T, L}$, all $\Delta \mathcal{O}_{k, n}^{T, L}$ show the same decay as they can be generated by $n-1$ derivatives with respect to $m_{k}^{2}$ from $\Delta \mathcal{O}_{k, 1}^{T, L}$.

The thermal decay at infinite volume and the volume decay at vanishing temperatures are given by the respective limits. For $L T \rightarrow \infty, \sqrt{75}$ gives the thermal decay (74) with $c_{T}=c_{T}(\infty)$, that is

$$
\lim _{k / T \rightarrow \infty}\left|\Delta \mathcal{O}_{k}^{T, \infty}\right| \propto A\left(\frac{k}{T}, \infty\right) \exp \left(-c_{T} \frac{k}{T}\right),
$$

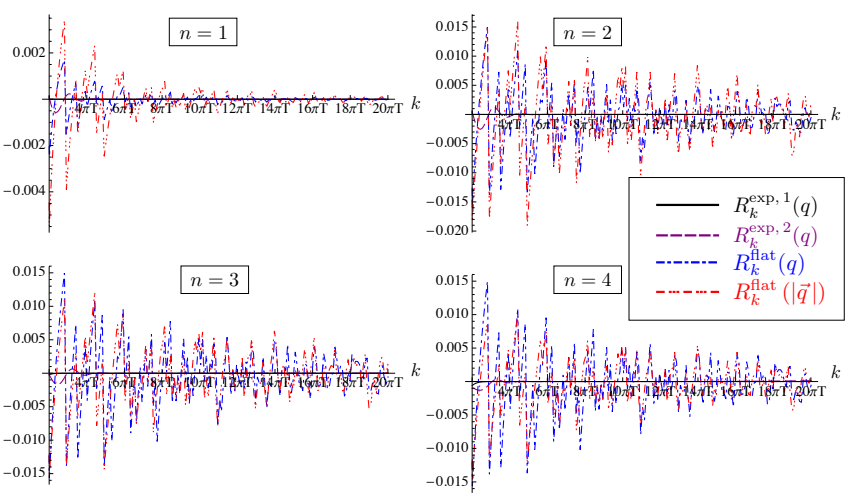

FIG. 9: Flows given by for $n=1,2,3,4$ in a finite volume for different regulators.

see Fig. 7, Fig. 8 . This is the analogue of $(30)$ with the substitution (74). In turn, for $L T \rightarrow 0$, 75 describes the volume decay at vanishing temperature, and we have

$$
\lim _{L T \rightarrow 0} \frac{c_{T}(L T)}{L T}=c_{L},
$$

and the equivalent relation to the purely thermal decay, 79 for the pure finite volume case,

$$
\lim _{L k \rightarrow \infty}\left|\Delta \mathcal{O}_{k}(0, L)\right| \propto A(\infty, k L) \exp \left(-c_{L} k L\right) .
$$

The function $c_{T}(L T)$ interpolates between the asymptotic thermal scaling and the asymptotic finite volume scaling, and depends on the shape of the regulator, $c_{T}(L T)=c_{T}\left(R_{k} ; L T\right)$. It can be extracted in the limit of $k \rightarrow \infty$ by

$$
c_{T}(L T)=-\frac{T}{k} \lim _{k \rightarrow \infty} \log \left\{\left|\Delta \mathcal{O}_{k}(T, L)\right|\right\} .
$$

In Fig. 10 we study the approach of the right hand side of (82) towards $c_{T}$ for $n=1,2,3,4$ and an exponential regulator, (4), with $m=2$, in both the infinite and finite volume case, in the latter case we use $L=1 / T$. The local minima indicate the local maxima and minima in the positive and negative envelopes of the decay, as seen in Fig. 3 and Fig. 4 for $n=1$. We find that the asymptotic limits for $k / T$ and/or $k /(1 / L)$ are reached quickly above the characteristic scale induced by $c_{T}$.

In Fig. 11 we study the interpolation of $c_{T}$ between the asymptotic forms at $T=0$ and $L=\infty$ for the exponential regulators with $n=1,2$. For this figure we have extracted $c_{T}$ with the value of the minimum at maximal $k$, in e.g. Fig. 10 the minimum between $8 \pi T$ and $10 \pi T$. We see that the interpolation regime at about $L T \approx 1$ is rather small and the asymptotic values are approached rapidly. 


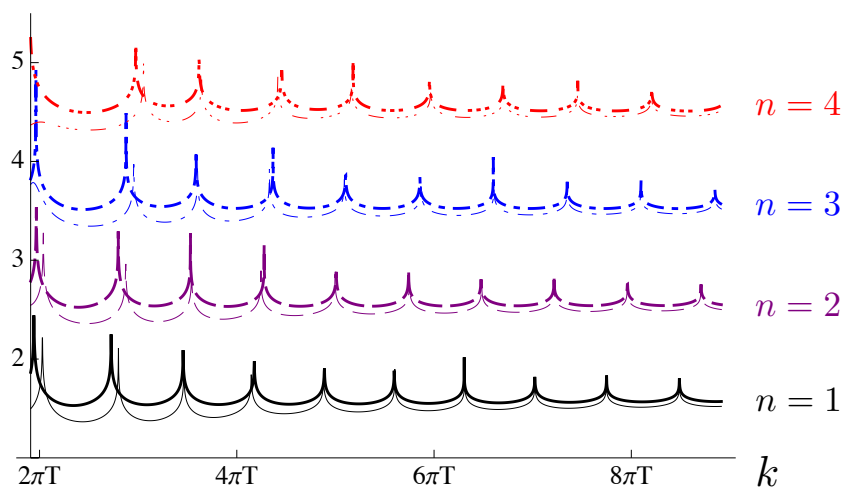

FIG. 10: Approach of flows towards the vacuum limit for scales $k$ larger the temperature and volume as parametrised by (82) and the an exponential regulator, (4), with $m=2$. Thick (thin, lower) curves are in infinite (finite with $L=1 / T$ ) volume.

\section{B. Thermal \& volume dependence of the couplings}

The results obtained in the last Sections exemplified the thermal and finite volume decays at the example of the thermodynamic observables, the free energy density and the pressure. Both observables have power counting dimension four, and indeed their flows scale with $k^{4}$ for non-analytic regulators. This leads to non-trivial initial conditions for these observables at the initial large cutoff scale $k=\Lambda$. We expect only power-counting reduced problems for the flow of the vertices, in particular for the mass $m_{k}^{2}$ and the coupling $\lambda_{k}$. If this reduction leads to sufficiently strong suppression in powers of $1 / k$ even nonanalytic regulators would allow for a direct access to both the thermodynamics as well as the flow of the couplings without resorting to non-trivial initial conditions at least beyond leading order.

As for the thermodynamics it is illustrative to simply consider the length derivative of the flows. For the effective potential this can be directly read off from 72 , 73 ,

$$
L \frac{\partial \dot{V}_{k}}{\partial L}=-\frac{1}{2} \S_{q}\left(2 \vec{q}^{2} \partial_{\vec{q}^{2}}-L \frac{\partial m_{k}^{2}}{\partial L} \partial_{m_{k}^{2}}\right) G_{k} \dot{R}_{k}
$$

The second term in 83 can be viewed as an improvement term. It does not dominate the flow. For the present purpose of a power counting analysis we drop it and concentrate on the first term. The flow of the effective potential reads for the three-dimensional and four-dimensional flat regulators,

$$
L \frac{\partial \dot{V}_{k}^{\text {3dflat }}(\rho)}{\partial L} \propto \frac{k^{3}}{L^{3}} \sum_{n_{i} \in \mathbb{Z}} \frac{\operatorname{coth} \frac{k}{2 T} \sqrt{1+\frac{m_{k}^{2}(\rho)}{k^{2}}}}{\sqrt{1+\frac{m_{k}^{2}(\rho)}{k^{2}}}} \delta\left(k^{2}-\vec{q}^{2}\right),
$$

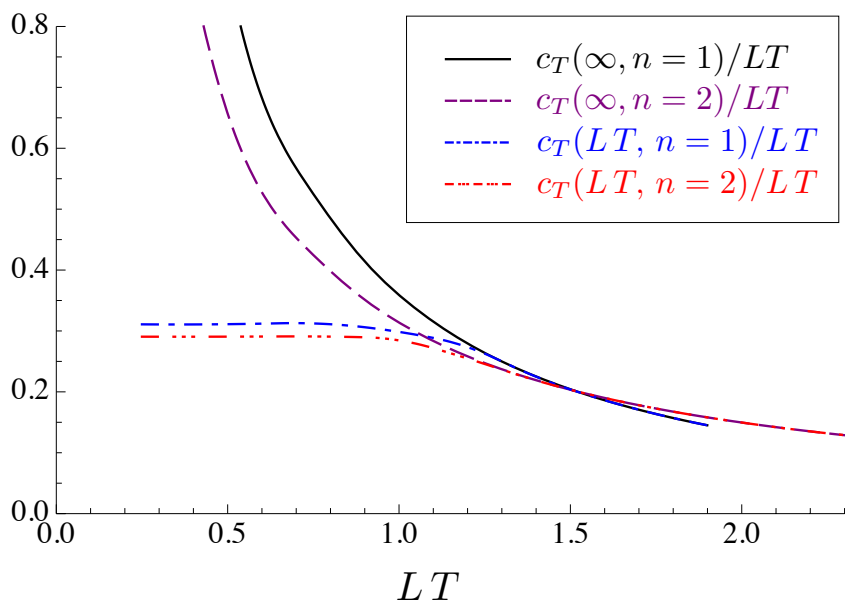

FIG. 11: Interpolation of $c_{T}$ for an exponential regulator, 4, with $m=2$ from the asymptotic purely thermal decay to the asymptotic purely finite volume decay.

and

$$
L \frac{\partial \dot{V}_{k}^{4 \text { dflat }}(\rho)}{\partial L} \propto 2 \frac{T}{L^{3}} \sum_{n_{\mu} \in \mathbb{Z}} \frac{\vec{q}^{2}}{1+\frac{m_{k}^{2}(\rho)}{k^{2}}} \delta\left(k^{2}-q^{2}\right)
$$

respectively, where the superscripts indicate the chosen regulator. The question of the sufficient decay that is at the heart of $T$ and $L$-independent initial conditions can already be answered at one loop. Thus, we integrate the flows in (85) at one loop from $k=0$ to $\Lambda$. This simply removed the $\delta$-function leading to

$$
\begin{aligned}
& V_{\Lambda}^{\text {3dflat }}(\rho)-V_{0}^{\text {3dflat }}(\rho) \propto \frac{1}{6} \frac{\Lambda}{L^{3}} \sum_{\vec{q}^{2} \leq \Lambda^{2}} \frac{\operatorname{coth} \frac{\Lambda}{2 T} \sqrt{1+\frac{m_{\Lambda}^{2}(\rho)}{\Lambda^{2}}}}{\sqrt{1+\frac{m_{\Lambda}^{2}(\rho)}{\Lambda^{2}}}}, \\
& V_{\Lambda}^{\text {4dflat }}(\rho)-V_{0}^{\text {4fflat }}(\rho) \propto \frac{1}{3} \frac{T}{L^{3}} \sum_{q^{2} \leq \Lambda^{2}} \frac{\vec{q}^{2} / \Lambda^{2}}{1+\frac{m_{\Lambda}^{2}(\rho)}{\Lambda^{2}}},
\end{aligned}
$$

where we have also integrated over the box size from infinite length to $L$. The expressions for the one loop couplings $m_{\Lambda}^{2}$ and $\lambda_{\Lambda}$ are derived from $(86)$ by two and four derivatives with respect to $\phi$, respectively. This involves terms that are proportional to the first and second derivative of 86 with respect to $m_{\Lambda}^{2}$, respectively. Hence, we have additional suppressions with $1 / \Lambda^{2}$ and $1 / \Lambda^{4}$, respectively. In turn, the sums in 86 grow proportional to $\Lambda^{3}$ and $\Lambda^{4}$ on the average. Sweeping over a single discrete frequency and spatial momentum value leads to a jump of the effective potentials $V_{\Lambda}^{3 \text { dflat }}$ and $V_{\Lambda}^{\text {4dflat }}$ with a height proportional to $\Lambda$ and $\Lambda^{0}$ respectively. For the couplings this entails a suppression of the single jumps with $1 / \Lambda$ and $1 / \Lambda^{2}$ for $m_{\Lambda}^{2}$, and $1 / \Lambda^{3}$ and $1 / \Lambda^{4}$ for $\lambda_{\Lambda}$.

In the following, we restrict ourselves to the efficient regulator of the form (4) with $m=1$. For this choice the transition to the vacuum theory happens at cutoff 


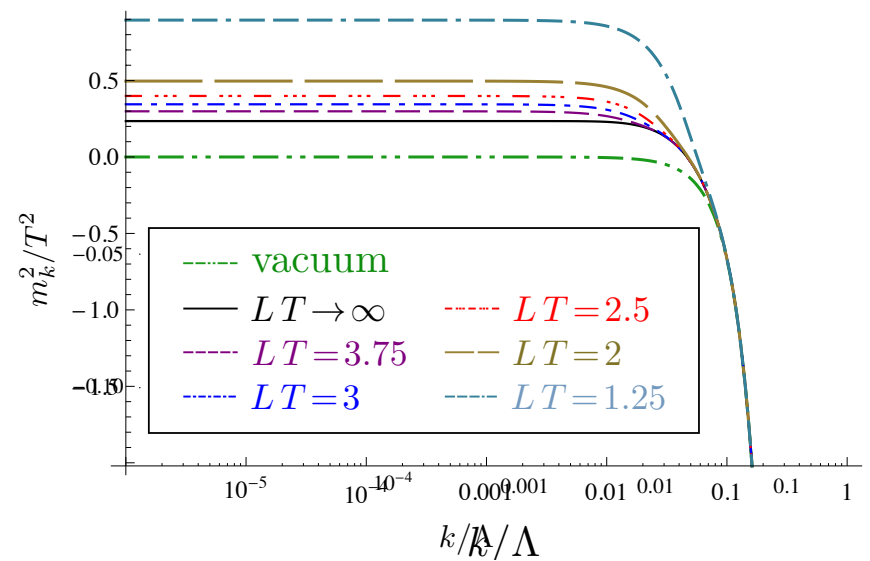

FIG. 12: Thermal and finite volume dependence of the mass for large UV coupling and $T / \Lambda=1 / 40$, and the exponential regulator, (4) with $m=1$.

scales close to the first frequency, and the cutoff scale can indeed be identified with a mass scale $m_{\text {gap }}$ of the order of the cutoff scale $k$.

In principle, there are three different scales in the problem at hand: vacuum mass, temperature and volume. In order to study the interplay of temperature and finite volume only, we consider the massless theory in the vacuum. Note that this implies in general $m_{k}^{2} \neq 0$ for cutoff scale $k \neq 0$. The vanishing mass at $k \rightarrow 0$ requires a fine tuning at $k=\Lambda$ : for a given initial coupling $\lambda_{\Lambda}$ we vary the initial value of the mass $m_{\Lambda}$ such that $m_{k \rightarrow 0}=0$. A generalisation to finite masses is straightforward but not the main purpose of this work.

In summary, from the value $m_{k=0}$ we generate the initial condition $m_{\Lambda}$ and $\lambda_{\Lambda}$ for mass and coupling flows, (24) with 29), to be solved in a self-consistent way. The functional behaviour of the coupling and, consequently, of the mass is significantly different for large and small values of $\lambda_{\Lambda}$. For large initial values of the coupling there are strong changes in mass and coupling, i.e. the running is very strong. For smaller values of the coupling the running is slowed down. This results in the fact that the couplings at $k=0$ are much closer to each other than at the initial scale. This behaviour is of course well know and explained by the fact, that the coupling appears quadratically and cubically, respectively, as a prefactor in the flows of the mass and coupling.

The effects of thermal fluctuations have been studied extensively in e.g. [43, 44, for the $\phi^{4}$-theory, for YangMills theory this has been done in [6] 8 : As finite temperature is an infrared modification of the theory, the theory is unchanged at scales larger than the typical temperature scale, which is set by the first Matsubara frequency $2 \pi T$. Hence, above this scale the flow follows the vacuum flow. Below this scale, temperature induces additional fluctuations which accelerate the flow. For the mass this results in the generation of a thermal mass in addition to the vacuum mass. However, once this generated scale is

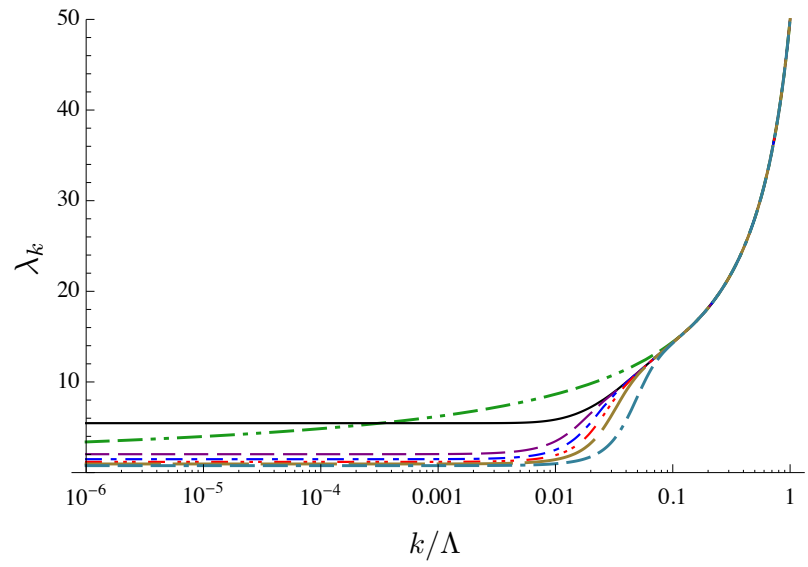

FIG. 13: Thermal and finite volume dependence for the coupling for large UV coupling. Regulator, parameters and colour-coding are the same as in Fig. 12

reached the mass suppresses fluctuation in both flows of the mass and the coupling. As a consequence, the flows are frozen below this scale and the values of the coupling and mass saturate.

In this work, we consider the thermal field theory in a finite volume. Similar to the compactification of the temporal direction due to non-zero temperature, the finite edge length of the (cubic) $3 d$-box is a modification of the infrared. Therefore, its effect is qualitatively similar to thermal effects.

Once the theory is probed at distances comparable to the volume fluctuations become stronger and generate an additional mass. At some point, however, the flows stop as fluctuations are suppressed by the mass. In general, it depends on the magnitude of the three scales $L, T$ and $m_{0}$, as already the largest scale effectively suppresses long range fluctuations and leads to an independence of further macroscopic details. Having tuned to $m_{0}=0$, the only relevant parameter that determines the behaviour of the theory is the ratio of spatial length $L$ to temporal length $1 / T$, hence $L T$. Thus, as long as cutoff effects are correctly taken care of, i.e. $\Lambda \gg 2 \pi T$ and $\Lambda \gg 2 \pi / L$, see discussion above, different combinations of $L$ and $T$ give similar results as long as their product is kept constant. In other words, the effects of finite volume are always relative to the temperature scale and it is thus sufficient to study only one temperature for different lengths.

In Fig. 12 Fig. 13 we show cutoff scale dependence of the mass and the coupling for large initial values of the coupling. In Fig. 14 and Fig. 15 we show the cutoff scale dependence for small initial couplings. Note that the flows are damped by $k^{2}$, unlike the flow of the potential. Thus, the technical problem of "mode-counting" cutoff does affect the mass or coupling but becomes only relevant at the level of the potential. It is clearly visible 


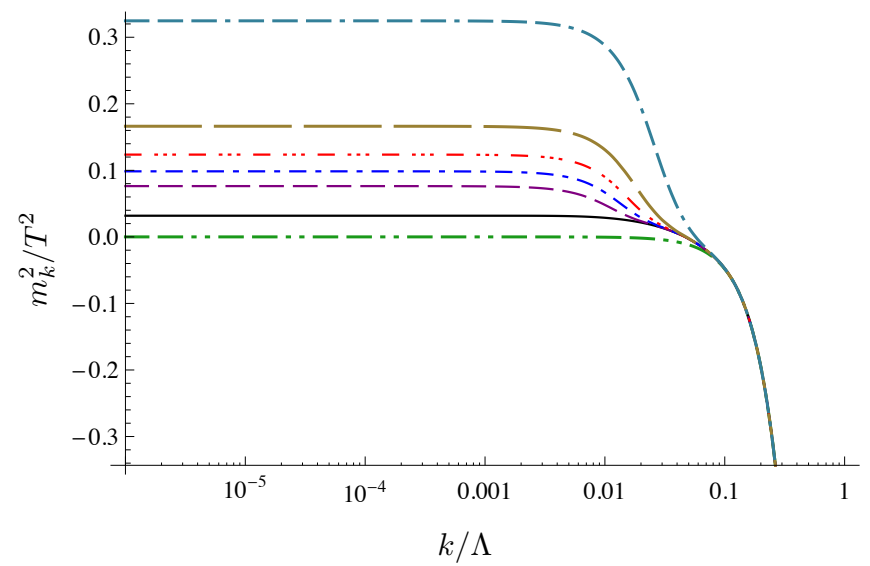

FIG. 14: Thermal and finite volume effects in the mass for small UV coupling. Regulator, parameters and colour-coding are the same as in Fig. 12 but the coupling from Fig. 15

that the effect of the finite volume is qualitatively equal to the effect of finite temperature for both the mass, cf. Fig. 12 and Fig. 14, and the coupling, cf. Fig. 13 and Fig. 15. Furthermore, the dependence on the finite volume is monotonic in the sense that the larger the volume the less additional flow is generated, i.e. the infinite volume limit is approached. Interestingly, the limit of infinite volume is approached very slowly, however, i.e. the value of $L T \gg 1$. This is due to the fact that the finite volume affects three dimensions and is to be compared with the compactification of the temporal direction only. The mass flows for a massless and massive theory are shown in Fig. 16, which exemplifies that the vacuum mass does not change the effect of finite temperature of volume qualitatively. This justifies our choice $m_{0}=0$.

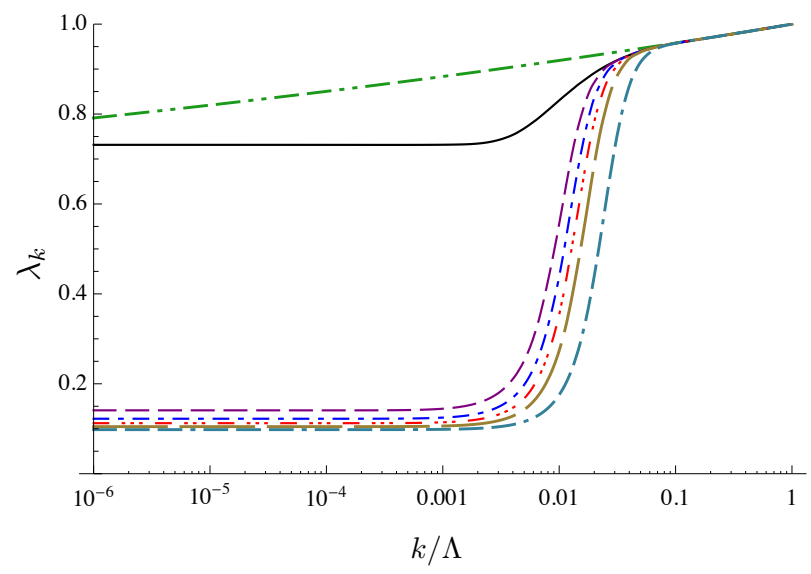

FIG. 15: Thermal and finite volume effects in the coupling for small UV coupling. Regulator, parameters and colour-coding are the same as in Fig. 14 .

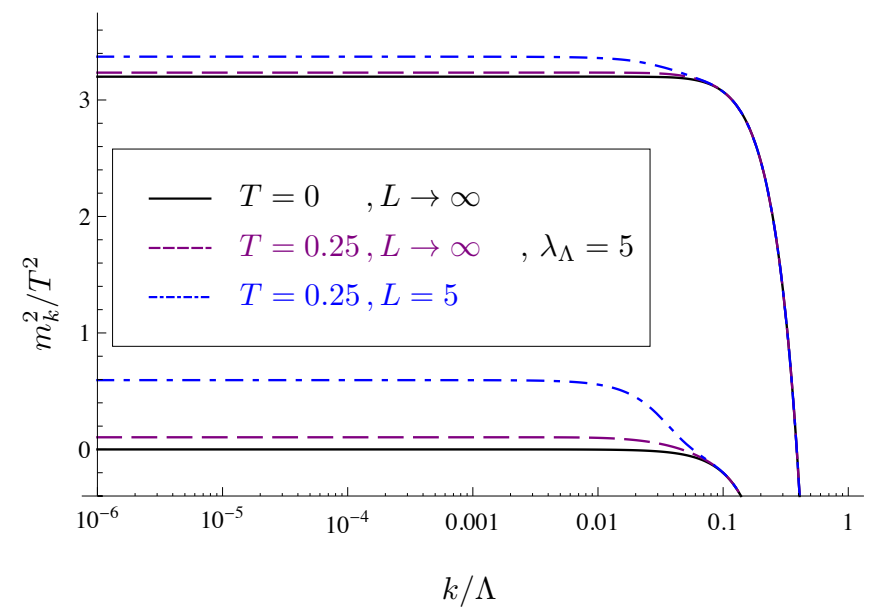

FIG. 16: Temperature and volume dependence of the mass for a massless (lower curves) and massive (upper curves) vacuum theory with $m_{k=0}^{2}=0.2$ for $\lambda_{\Lambda}=5$ at the initial scale.

In Fig. 17] we show the dimensionless mass as a function of the value of the coupling at $2 \pi T$. We choose this value to facilitate comparison with other approaches where the coupling is defined at that scale, e.g. perturbation theory. Again, finite volume effects strengthen fluctuations and the effective mass is therefore increased. Thus, the infinite volume limit for the mass is approached from above. This is similar to the well-known thermal behaviour, see Fig. 18 .

Eventually, we consider the pressure. Note again that is can be evaluated trivially by integrating the flow equation (33) with the mass function computed above. In Fig. 2 the flow of the pressure is shown. Integrating the flow for a trivial initial conditions gives the pressure. In Fig. 19 the pressure is shown as a function of the value of the coupling at $2 \pi T$ and normalised to the Stefan-Boltzmann pressure. The infinite volume case

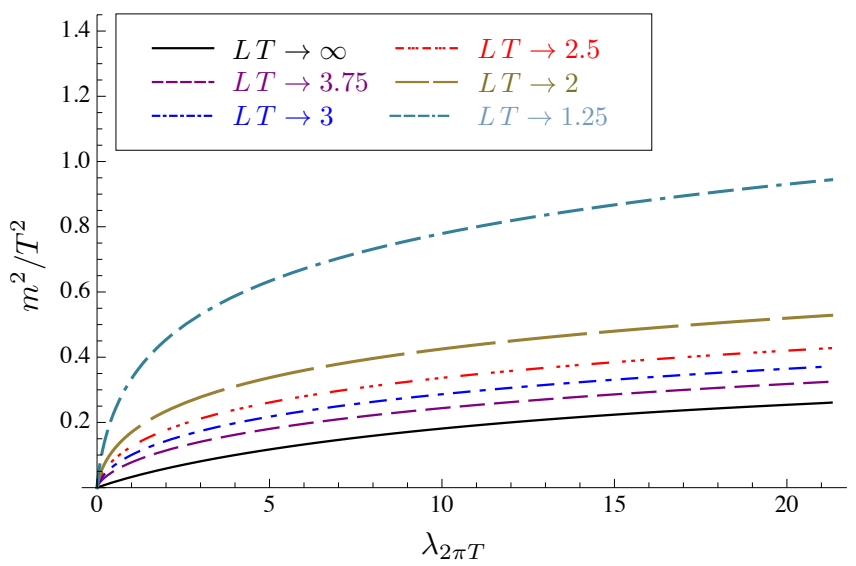

FIG. 17: Masses as a function of the coupling at $2 \pi T$ for different volumes. 


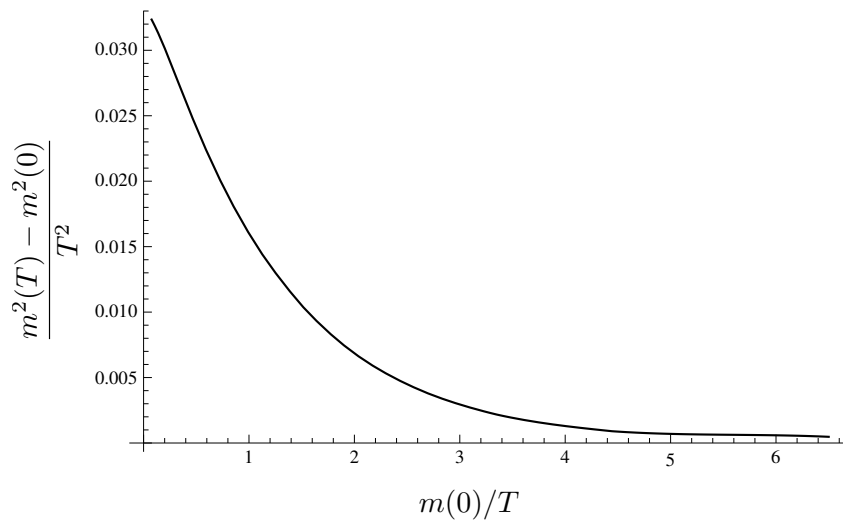

FIG. 18: Mass ratio of thermal and vacuum mass as a function of (fixed) vacuum mass over temperature. The ratio shows an exponential decay with $\exp -m(0) / T$.

agrees with results in the literature. We see that both, increasing the coupling and decreasing the volume leads to a smaller pressure. Moreover, in comparison to the free energy density the thermal pressure shows significantly less volume dependence. This reflects the fact that it accounts for thermal fluctuations and their volumedependence.

\section{CONCLUSIONS}

We have investigated finite volume effects for the thermodynamics of a $\phi^{4}$ theory within the functional renormalisation group approach. We have focused on conceptual properties of the regulator dependence of flows in finite volume compared to infinite volume. The applicability of regulators can be decreased or even spoiled by the non-analyticity of the regulator for momenta at the RG scale, $p^{2}=k^{2}$. We find that the thermal flow of the effective action exhibits oscillations along $k$. Their range becomes larger with the sharpness of the regulator. In the limit of a sharp or flat regulator these oscillations extend to infinity. For a massless theory the only scales present are the temperature and the spatial length. Hence, effectively the theory only depends on one parameter, the ratio of lengths in spatial to temporal directions, $L T$, where $L$ is the edge length of a cubic box, and $1 / T$ is the length in temporal direction.

The cutoff scale introduces an additional scale to the system and both length scales are visible as onset scales for thermal and finite volume effects respectively. For analytic regulators the onset scales are proportional to $T$ and $L$, while the proportionality constant depends on the chosen regulator. Above the onset scales the flows tend exponentially towards the corresponding vacuum flows.

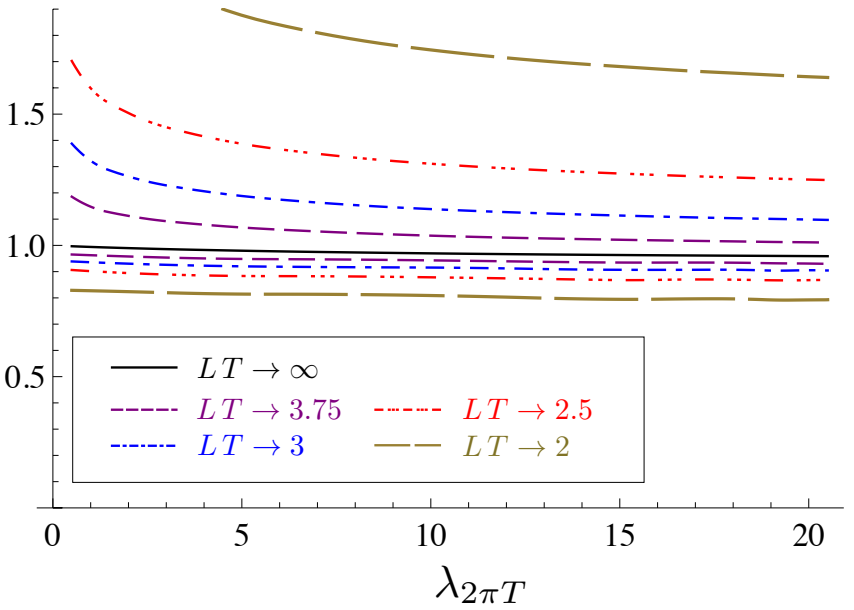

FIG. 19: Pressure, $p$, of $\phi^{4}$-theory in an infinite volume (black curve) and a finite volume (curves below infinite volume result) in comparison with the free energy, $-f_{k=0}$ (curves above infinite volume result).

In turn, for non-analytic regulators the exponential decay towards the vacuum flows is missing, which makes quantitative studies of thermal and finite volume effects cumbersome.

As expected, both finite volume and thermal effects show similar dependences on the mass scales of the theory including the cutoff scale. This is directly seen in volume dependence of the thermal mass already, where the mass is larger for smaller volumes. As a consequence, the infinite volume limit of the mass is reached from above.

Furthermore, we have studied the pressure. The infinite volume limit is reached quicker for larger couplings, because correlations are transferred in a more efficient way and fluctuations are washed out before the length scale is reached. Therefore, for smaller couplings the effects of the finite length is stronger. This is again a manifestation that these are pure infrared effects.

The structural aspects of the finite volume dependence studied in the present work are quite general. The present findings carry over straightforwardly to $O(N)$ models with more than one field component, to phenomenological low energy systems such as the quark-meson model, and, to some extend to full QCD. In the latter case the gauge sector requires some special attention. This will be studied elsewhere.

Acknowledgments - We thank J. Braun and B.J. Schaefer for discussions. LF is supported by ERC-AD-267258. Moreover, this work is supported by the Helmholtz Alliance HA216/EMMI and by ERCAdG-290623. 


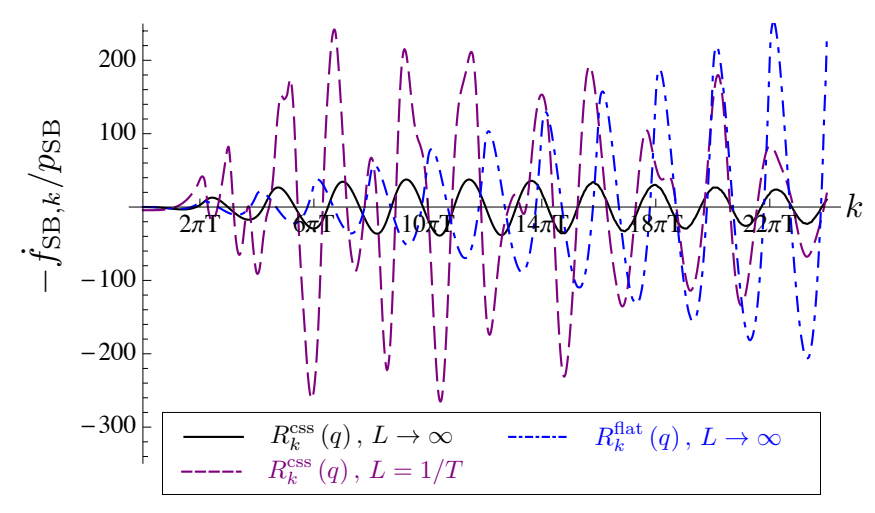

FIG. 20: Flow of the Stefan-Boltzmann free energy $\dot{f}_{\mathrm{SB}, k}$ for the css regulators (B1) in finite and infinite volume compared to the flat regulator (5), in the infinite volume.

\section{Appendix A: Pressure flows for a flat box cutoff}

For a fully analytical approach we choose the product cutoff

$$
R_{k}^{\mathrm{prod}}(q)=\prod_{\mu=0}^{4} R_{k}^{\mathrm{flat}}\left(q_{\mu}\right)
$$

with

$$
G_{k}^{\mathrm{prod}}(q)=\frac{1}{k^{2}} \prod_{\mu=0}^{4} \theta\left(k^{2}-q_{\mu}^{2}\right)+\frac{1}{q^{2}}\left[1-\prod_{\mu=0}^{4} \theta\left(k^{2}-q_{\mu}^{2}\right)\right]
$$

The flow of $f$ is then given by

$$
\dot{f}_{\mathrm{SB}, k}=\frac{T}{\mathcal{V}}\left\{\left(2 N_{T}+1\right)\left(\frac{N_{L}}{3}+N_{L}^{2}+\frac{2 N_{L}^{3}}{3}\right)^{3}+3\left(2 N_{L}+1\right)\left(\frac{N_{T}}{3}+N_{T}^{2}+\frac{2 N_{T}^{3}}{3}\right)\left(\frac{N_{L}}{3}+N_{L}^{2}+\frac{2 N_{L}^{3}}{3}\right)^{2}\right\}-\frac{8 k^{4}}{27 \pi^{4}}
$$

with

$$
N_{L}=\left[\frac{k L}{2 \pi}\right], \quad N_{T}=\left[\frac{k}{2 \pi T}\right],
$$

where the brackets indicate the largest integer number smaller than the argument. The enveloping function of (A3) rising as $k^{3}$. This exemplifies the problem related to non-analytic regulators.

\section{Appendix B: Compactly supported smooth regulator}

Here we test the decay for compactly supported smooth (css) regulators suggested in [42],

$$
\begin{aligned}
R_{k}^{\mathrm{css}}(q) & =q^{2} r_{k}^{\mathrm{css}}\left(q^{2} / k^{2}\right), \\
r_{k}^{\mathrm{css}}(y) & =\frac{\exp \left\{c y_{0}^{b} /\left(1-y_{0}^{b}\right)-1\right\}}{\exp \left\{c y^{b} /\left(1-y^{b}\right)-1\right\}} \Theta(1-y),
\end{aligned}
$$

with the normalisation $y_{0}$ such that $r_{k}^{\mathrm{css}}\left(y_{0}\right) \equiv 1$. We choose the parameters $b=1, c=1$ and $y_{0}=1 / 2$ leading to a standard exponential decay. In Fig. 20 we show the flow of the Stefan-Boltzmann free energy $f_{\mathrm{SB}, k}$ for the css regulators (B1) in comparison to the flat regulator (5). In both cases of infinite and finite volume $\dot{f}_{\mathrm{SB}, k}$ does not show the exponential decay for large cutoff scales $k$.

\section{Appendix C: Flows for the $L$-derivatives of the couplings}

In the present $\phi^{4}$-approximation to the LPA one has to compute $m_{k}^{2}(L)$ and $\lambda_{k}(L)$. More generally, in a polynomial expansion of the LPA, where

$$
\vec{\lambda}=\left(\lambda_{1}, \lambda_{2}, \lambda_{3}, \ldots\right), \quad \text { with } \quad \lambda_{1}=\kappa, \quad \lambda_{2}=\lambda,
$$

there are additional couplings. Their flows are given schematically by

$$
\dot{\kappa}=-\frac{1}{2} \int_{q} G_{k}\left(q, \rho_{0}\right) \dot{R}_{k}(q)
$$

and

$$
\dot{\lambda}_{n}-\dot{\kappa} \lambda_{n+1}=\frac{1}{2} \int_{q} G_{k}^{(n)}\left(q, \rho_{0}\right) \dot{R}_{k}(q),
$$

where $G^{(n)}(q, \rho)=\partial_{\rho}^{n} G(q, \rho)$, using 15 . Taking the $L$ derivative (or $\mathcal{V}$-derivative) of $\mathrm{C} 3$, with

$$
\lambda_{n}^{\prime}=L \partial_{L} \lambda_{n}=3 \mathcal{V} \frac{\partial \lambda_{n}}{\partial \mathcal{V}},
$$

leads to flow equations for the $L$-derivatives of $\lambda_{n}$, therefore,

$$
\partial_{t} \kappa^{\prime}=-\frac{1}{2} \int_{q}\left(2 \vec{q}^{2} \partial_{\vec{q}^{2}}-\lambda_{i}^{\prime} \partial_{\lambda_{i}}\right) G_{k}^{2}\left(q, \rho_{0}\right) \dot{R}_{k}(q),
$$


and

$$
\begin{aligned}
& \partial_{t} \lambda_{n}^{\prime}-\partial_{t} \lambda_{1}^{\prime} \lambda_{n+1}+\partial_{t} \lambda_{1} \lambda_{n+1}^{\prime} \\
& \quad=\quad-\frac{1}{2} \int_{q}\left(2 \vec{q}^{2} \partial_{\vec{q}^{2}}-\lambda_{i}^{\prime} \partial_{\lambda_{i}}\right) G^{(n)}\left(q, \rho_{0}\right) \dot{R}_{k}(q) .
\end{aligned}
$$

Note that $\rho_{0}=\max (0, \kappa)$ depends on $\lambda_{1}=\kappa$. Eqs. C5, (C6) can be solved on the basis of a solution for $\lambda_{k}$ in a given volume $\mathcal{V}$ (a cube with box length $L$ ), and a regulator that guarantees the decay of volume fluctuations for $k \rightarrow \infty$, that is

$$
\lim _{k \rightarrow \infty} \lambda_{n}^{\prime}=0 .
$$

Eq. (C7) requires sufficiently smooth regulators in the spatial momentum directions. Moreover, iterations of this procedure leads to flow equations of $N$ th $L$ derivatives of the couplings $\vec{\lambda}$ in the basis of $j=0, \ldots, N-1$ $L$-derivatives of $\vec{\lambda}$ and their flows.

The present derivations trivially extend to the situation of different spatial lengths $L_{i}$ for $i=1,2,3$. We add that the present procedure applies more generally to flows of derivatives with respect to external parameters such as volume, temperature, chemical potential, and couplings.
[1] J. Braun, B. Klein, and H. Pirner, Phys.Rev. D72, 034017 (2005), hep-ph/0504127.

[2] J. Braun, B. Klein, and P. Piasecki, Eur.Phys.J. C71, 1576 (2011), 1008.2155.

[3] J. Braun, B. Klein, and B.-J. Schaefer, Phys.Lett. B713, 216 (2012), 1110.0849.

[4] J. Braun, S. Diehl, and M. M. Scherer (2011), 1109.1946.

[5] R.-A. Tripolt, J. Braun, B. Klein, and B.-J. Schaefer, Phys.Rev. D90, 054012 (2014), 1308.0164.

[6] L. Fister and J. M. Pawlowski (2011), 1112.5440.

[7] L. Fister and J. M. Pawlowski, PoS QCD-TNT-II, 021 (2011), 1112.5429.

[8] L. Fister, doctoral thesis, Heidelberg University, (2012), 2012.

[9] L. Fister and J. M. Pawlowski, Phys.Rev. D88, 045010 (2013), 1301.4163.

[10] L. Fister and J. M. Pawlowski (2013), 1302.1373.

[11] C. S. Fischer, A. Maas, J. M. Pawlowski, and L. von Smekal, Annals Phys. 322, 2916 (2007), hep-ph/0701050.

[12] J. Luecker, C. S. Fischer, and R. Williams, Phys.Rev. D81, 094005 (2010), 0912.3686.

[13] J. A. Bonnet, C. S. Fischer, and R. Williams, Phys.Rev. B84, 024520 (2011), 1103.1578.

[14] J. A. Bonnet and C. S. Fischer, Phys.Lett. B718, 532 (2012), 1201.6139.

[15] C. Wetterich, Phys.Lett. B301, 90 (1993).

[16] D. F. Litim, Phys.Lett. B486, 92 (2000), hepth/0005245.

[17] D. F. Litim, Phys.Rev. D64, 105007 (2001), hepth/0103195.

[18] J. M. Pawlowski, Annals Phys. 322, 2831 (2007), hepth/0512261.

[19] J. M. Pawlowski and F. Rennecke, Phys. Rev. D 90, 076002 (2014), 1403.1179.

[20] O. Bohr, B.-J. Schaefer, and J. Wambach, Int.J.Mod.Phys. A16, 3823 (2001), hep-ph/0007098.

[21] D. F. Litim, JHEP 0111, 059 (2001), hep-th/0111159.

[22] D. F. Litim, Nucl.Phys. B631, 128 (2002), hepth/0203006.

[23] D. F. Litim and D. Zappala, Phys.Rev. D83, 085009
(2011), 1009.1948.

[24] J. Borchardt and B. Knorr (2015), 1502.07511.

[25] S. Tan, Annals Phys. 323, 2952 (2008), condmat/0505200.

[26] S. Tan, Annals Phys. 323, 2971 (2008), condmat/0508320.

[27] S. Tan, Annals Phys. 323, 2987 (2008), 0803.0841.

[28] I. Boettcher, S. Diehl, J. M. Pawlowski, and C. Wetterich (2012), 1209.5641.

[29] J. Keitel and L. Bartosch, J.Phys. A45, 105401 (2012), 1109.3013.

[30] P. Jakubczyk and M. Napiorkowski, Phys.Rev. B87, 165439 (2013), 1212.2647.

[31] D. F. Litim and J. M. Pawlowski, pp. 168-185 (1998), hep-th/9901063.

[32] D. F. Litim and J. M. Pawlowski, JHEP 0611, 026 (2006), hep-th/0609122.

[33] D. R. Heath-Brown, in Number theory in progress, Proc. Number Theory Conf. Zakopane, eds. K. Györy et al., Vol. 2, 883 (1997).

[34] M. N. Huxley, in Number Theory for the Millennium, ed. A. K. Peters, Vol II, 275 (2002).

[35] V. Skokov, Phys.Rev. D85, 034026 (2012), 1112.5137.

[36] K. Fukushima and J. M. Pawlowski, Phys.Rev. D86, 076013 (2012), 1203.4330.

[37] J. O. Andersen, W. R. Naylor, and A. Tranberg, JHEP 1502, 042 (2015), 1410.5247.

[38] J. Braun, W. A. Mian, and S. Rechenberger (2014), 1412.6025 .

[39] N. Mueller and J. M. Pawlowski (2015), 1502.08011.

[40] J. O. Andersen, W. R. Naylor, and A. Tranberg (2014), 1411.7176.

[41] V. A. Miransky and I. A. Shovkovy, Phys.Rept. 576, 1 (2015), 1503.00732.

[42] I. Nandori, JHEP 1304, 150 (2013), 1208.5021.

[43] J.-P. Blaizot, A. Ipp, R. Mendez-Galain, and N. Wschebor, Nucl.Phys. A784, 376 (2007), hep-ph/0610004.

[44] J.-P. Blaizot, A. Ipp, and N. Wschebor, Nucl.Phys. A849, 165 (2011), 1007.0991. 